\title{
Article
}

\section{COVLIAS 1.0: Lung Segmentation in COVID-19 Computed Tomography Scans Using Hybrid Deep Learning Artificial Intelligence Models}

\author{
Jasjit S. Suri ${ }^{1,2, *}$, Sushant Agarwal ${ }^{2,3}{ }^{\oplus}$, Rajesh Pathak ${ }^{4}$, Vedmanvitha Ketireddy ${ }^{5}$, Marta Columbu ${ }^{6}$, Luca Saba ${ }^{6}$, \\ Suneet K. Gupta ${ }^{7}$, Gavino Faa ${ }^{8}$, Inder M. Singh ${ }^{1}$, Monika Turk ${ }^{9}$, Paramjit S. Chadha ${ }^{1}$, Amer M. Johri ${ }^{10}$, \\ Narendra N. Khanna ${ }^{11}$, Klaudija Viskovic ${ }^{12}$, Sophie Mavrogeni ${ }^{13}$, John R. Laird ${ }^{14}$, Gyan Pareek ${ }^{15}$, \\ Martin Miner ${ }^{16}$, David W. Sobel ${ }^{15}$, Antonella Balestrieri ${ }^{6}$, Petros P. Sfikakis ${ }^{17}$, George Tsoulfas ${ }^{18}$ (D), \\ Athanasios Protogerou ${ }^{19}{ }^{\circledR}$, Durga Prasanna Misra ${ }^{20}\left(\right.$, Vikas Agarwal ${ }^{20}\left({ }^{(0}\right.$, George D. Kitas ${ }^{21,22}$, Jagjit S. Teji ${ }^{23}$, \\ Mustafa Al-Maini ${ }^{24}$, Surinder K. Dhanjil ${ }^{25}$, Andrew Nicolaides $\left.{ }^{26}{ }^{(}\right)$, Aditya Sharma ${ }^{27}$, Vijay Rathore ${ }^{25}$, \\ Mostafa Fatemi ${ }^{28}$, Azra Alizad ${ }^{29}$ (D), Pudukode R. Krishnan ${ }^{30}$, Nagy Frence ${ }^{31}$, Zoltan Ruzsa ${ }^{31}$, Archna Gupta ${ }^{32}$, \\ Subbaram Naidu ${ }^{33}$ and Mannudeep Kalra ${ }^{34}$
}

check for updates

Citation: Suri, J.S.; Agarwal, S.; Pathak, R.; Ketireddy, V.; Columbu, M.; Saba, L.; Gupta, S.K.; Faa, G.; Singh, I.M.; Turk, M.; et al. COVLIAS 1.0: Lung Segmentation in COVID-19 Computed Tomography Scans Using Hybrid Deep Learning Artificial Intelligence Models. Diagnostics 2021, 11, 1405. https://doi.org/10.3390/ diagnostics11081405

Received: 6 July 2021

Accepted: 29 July 2021

Published: 4 August 2021

Publisher's Note: MDPI stays neutral with regard to jurisdictional claims in published maps and institutional affiliations.

Copyright: (c) 2021 by the authors. Licensee MDPI, Basel, Switzerland. This article is an open access article distributed under the terms and conditions of the Creative Commons Attribution (CC BY) license (https:// creativecommons.org/licenses/by/ $4.0 /)$.
1 Stroke Diagnostic and Monitoring Division, AtheroPoint ${ }^{\mathrm{TM}}$, Roseville, CA 95661, USA; drindersingh1@gmail.com (I.M.S.); pomchadha@gmail.com (P.S.C.)

2 Advanced Knowledge Engineering Centre, GBTI, Roseville, CA 95661, USA; sushant.ag09@gmail.com

3 Department of Computer Science Engineering, PSIT, Kanpur 209305, India

4 Department of Computer Science Engineering, Rawatpura Sarkar University, Raipur 492015, India; drrkpathak20@gmail.com

5 Mira Loma High School, Sacramento, CA 95821, USA; manvi.ketireddy@gmail.com

6 Department of Radiology, Azienda Ospedaliero Universitaria (A.O.U.), 09124 Cagliari, Italy; martagiuliacol@gmail.com (M.C.); lucasabamd@gmail.com (L.S.); antonellabalestrieri@hotmail.com (A.B.)

7 Department of Computer Science, Bennett University, Noida 201310, India; suneet.gupta@bennett.edu.in

Department of Pathology_AOU of Cagliari, 09124 Cagliari, Italy; gavinofaa@gmail.com

9 The Hanse-Wissenschaftskolleg Institute for Advanced Study, 27753 Delmenhorst, Germany; monika.turk84@gmail.com

10 Department of Medicine, Division of Cardiology, Queen's University, Kingston, ON K7L 3N6, Canada; johria@queensu.ca

11 Department of Cardiology, Indraprastha APOLLO Hospitals, New Delhi 208011, India; drnnkhanna@gmail.com

12 Department of Radiology, University Hospital for Infectious Diseases, 10000 Zagreb, Croatia; klaudija.viskovic@bfm.hr

13 Cardiology Clinic, Onassis Cardiac Surgery Center, 17674 Athens, Greece; soma13@otenet.gr

14 Heart and Vascular Institute, Adventist Health St. Helena, St. Helena, CA 94574, USA; Lairdjr@ah.org

15 Minimally Invasive Urology Institute, Brown University, Providence City, RI 02912, USA; gyan_pareek@brown.edu (G.P.); dwsobel@gmail.com (D.W.S.)

16 Men's Health Center, Miriam Hospital Providence, Providence, RI 02906, USA; martin_miner@brown.edu

17 Rheumatology Unit, National Kapodistrian University of Athens, 15772 Athens, Greece; psfikakis@med.uoa.gr

18 Department of Transplantation Surgery, Aristoteleion University of Thessaloniki, 54124 Thessaloniki, Greece; tsoulfasg@gmail.com

19 National \& Kapodistrian University of Athens, 15772 Athens, Greece; aprotog@med.uoa.gr

20 Department of Immunology, Sanjay Gandhi Postgraduate Institute of Medical Sciences, Lucknow 226014, India; durgapmisra@gmail.com (D.P.M.); vikasagr@yahoo.com (V.A.)

21 Academic Affairs, Dudley Group NHS Foundation Trust, Dudley DY1 2HQ, UK; george.kitas@nhs.net

22 Arthritis Research UK Epidemiology Unit, Manchester University, Manchester M13 9PL, UK

23 Ann and Robert H. Lurie Children's Hospital of Chicago, Chicago, IL 60611, USA; jteji@mercy-chicago.org

24 Allergy, Clinical Immunology and Rheumatology Institute, Toronto, ON M5G 1N8, Canada; almaini@hotmail.com

25 Athero Point LLC, Roseville, CA 95611, USA; surinderdhanjil@gmail.com (S.K.D.);

Vijay.s.rathore@kp.org (V.R.)

26 Vascular Screening and Diagnostic Centre, University of Nicosia Medical School, Nicosia 2408, Cyprus; anicolaides1@gmail.com

27 Division of Cardiovascular Medicine, University of Virginia, Charlottesville, VA 22904, USA; AS8AH@hscmail.mcc.virginia.edu 
28 Department of Physiology \& Biomedical Engg., Mayo Clinic College of Medicine and Science, Rochester, MN 55905, USA; fatemi.mostafa@mayo.edu

29 Department of Radiology, Mayo Clinic College of Medicine and Science, Rochester, MN 55905, USA; aza@mayo.edu

30 Neurology Department, Fortis Hospital, Bangalore 560076, India; prkrish12@rediffmail.com

31 Department of Internal Medicines, Invasive Cardiology Division, University of Szeged, 6720 Szeged, Hungary; drnagytfer@hotmail.com (N.F.); zruzsa@icloud.com (Z.R.)

32 Radiology Department, Sanjay Gandhi Postgraduate Institute of Medical Sciences, Lucknow 226014, India; garchna@gmail.com

33 Electrical Engineering Department, University of Minnesota, Duluth, MN 55455, USA; dsnaidu@d.umn.edu

34 Department of Radiology, Massachusetts General Hospital, 55 Fruit Street, Boston, MA 02114, USA; mkalra@mgh.harvard.edu

* Correspondence: jasjit.suri@atheropoint.com; Tel.: +1-(916)-749-5628

\begin{abstract}
Background: COVID-19 lung segmentation using Computed Tomography (CT) scans is important for the diagnosis of lung severity. The process of automated lung segmentation is challenging due to (a) CT radiation dosage and (b) ground-glass opacities caused by COVID-19. The lung segmentation methodologies proposed in 2020 were semi- or automated but not reliable, accurate, and user-friendly. The proposed study presents a COVID Lung Image Analysis System (COVLIAS 1.0, AtheroPoint ${ }^{\mathrm{TM}}$, Roseville, CA, USA) consisting of hybrid deep learning (HDL) models for lung segmentation. Methodology: The COVLIAS 1.0 consists of three methods based on solo deep learning (SDL) or hybrid deep learning (HDL). SegNet is proposed in the SDL category while VGG-SegNet and ResNet-SegNet are designed under the HDL paradigm. The three proposed AI approaches were benchmarked against the National Institute of Health (NIH)-based conventional segmentation model using fuzzy-connectedness. A cross-validation protocol with a 40:60 ratio between training and testing was designed, with $10 \%$ validation data. The ground truth (GT) was manually traced by a radiologist trained personnel. For performance evaluation, nine different criteria were selected to perform the evaluation of SDL or HDL lung segmentation regions and lungs long axis against GT. Results: Using the database of 5000 chest CT images (from 72 patients), COVLIAS 1.0 yielded AUC of $\sim 0.96, \sim 0.97, \sim 0.98$, and $\sim 0.96$ ( $p$-value $<0.001$ ), respectively within $5 \%$ range of GT area, for SegNet, VGG-SegNet, ResNet-SegNet, and NIH. The mean Figure of Merit using four models (left and right lung) was above $\mathbf{9 4 \%}$. On benchmarking against the National Institute of Health (NIH) segmentation method, the proposed model demonstrated a $58 \%$ and $44 \%$ improvement in ResNet-SegNet, 52\% and 36\% improvement in VGG-SegNet for lung area, and lung long axis, respectively. The PE statistics performance was in the following order: ResNet-SegNet $>$ VGG-SegNet $>$ NIH > SegNet. The HDL runs in $<1$ s on test data per image. Conclusions: The COVLIAS 1.0 system can be applied in real-time for radiology-based clinical settings.
\end{abstract}

Keywords: COVID-19; computed tomography; lungs; segmentation; hybrid deep learning

\title{
1. Introduction
}

On 30 January 2020, the International Health Regulations and Emergency Committee of the World Health Organization (WHO) declared COVID-19 or Coronavirus a "public health emergency of international concern" or "pandemic". As per the WHO's statistics dated 28 July 2021, more than 196 million people have been infected with Acute Respiratory Distress Syndrome (ARDS), and nearly 4.2 million have lost their lives due to this virus [1]. This SARS-CoV-2 virus affects the respiratory system, damages lungs, travels through the entire body, and causes myocardial infarction or coronary artery syndrome [2,3] or worsening diabetes [4] or causing pulmonary embolism [5]. It was seen that comorbidity had a severe influence on COVID-19 [6]. As of today, even though vaccination is showing the signs of pandemic control in some parts of the world, it is still the highest concern for health professionals. The objective is to flatten the curve and evaluate the COVID-19 lung severity at an early stage prior to worsening symptoms and admission of patients in ICU [7]. 
Computed Tomography (CT) is often used for assessing COVID-19 severity in the lung regions and is considered an important component of the computer-aided diagnosis for lung image analysis [7-9]. For a complete diagnosis of the COVID-19 severity, one must first identify the region of interest in these CT scans. There are two main challenges associated with the processing of CT scans: first and foremost, the challenge is the large volume of patients in diagnostic centers with each having 200 slices to be processed. This makes the task of processing scanned images tedious and time-consuming [10]. The second issue with current automated or semi-automated systems is reliability, accuracy, and clinical effectiveness [11]. One of the major causes for unreliable accuracy and low performance is the intensity-based segmentation methods which are influenced by local or global statistical methods. Furthermore, it does not take advantage of the cohort's knowledge. Thus, there is a clear need for an automated and accurate joint left and right lung identification system in CT scans.

Artificial Intelligence (AI) has provided fast pace research and development in nearly all walks of life especially in healthcare imaging [12]. The reason for such growth is the ability of AI techniques to mimic the brain using deep neural networks [12-14]. Machine learning is the most fundamental class of $\mathrm{AI}$ and has dominated the medical imaging industry [15], covering many fields of healthcare such as cancer classification and diagnosis of several kinds of cancers such as breast [16], liver [17,18], thyroid [19-21], skin [22,23], ovarian [24], and lung [25]. ML has also been applied to risk stratification for coronary artery disease [26,27], atherosclerosis imaging [28,29], and stroke [30], diabetes [31,32], and arrhythmia classification [33].

A subclass of ML is deep learning (DL), or conventionally called solo DL (SDL). Many researchers have proposed AI-based studies on COVD-19 which involves SDL techniques. [34,35]. It offers substantial benefits compared to ML-based solutions [11,36]. DL provides a complete automated feature extraction and classification simultaneously using the so-called dense layers. Despite the merits of DL, it poses challenges and uncertainties such as optimization of the learning rate, deciding the number of epochs, avoiding overfitting, handling large image size configurations, and operating in a multiresolution framework. One way to overcome challenges in SDL is by fusing two kinds of DL systems, called hybrid DL (HDL). It offers the advantages such as (i) extraction and refinement of a large number of features, (ii) application of diversity in classifiers, and (iii) ability to apply transfer learning for weights reusability. The proposed study uses the spirit of HDL to design COVID Lung Image Analysis System (COVLIAS) 1.0, which computes the segmentation of the left and right lungs jointly. The proposed framework uses two kinds of HDL models which combine Visual Geometry Group (VGG), Segmentation Network (SegNet), and Residual Network (ResNet) architectures. The model combines the strengths of both VGG and SegNet models and offers a new VGG-SegNet framework.

Similarly, Resnet-Segnet includes the power of both ResNet and SegNet. These models use 40:60 training to testing ratio protocol, then benchmarking our novel design against the NIH-based Fuzzy-Connectedness (FC) lung segmentation method [37]. Finally, we compute nine different ways for conducting the performance evaluation of the lung region and boundary estimation system.

The layout of this study is as follows: Section 2 presents the methodology with the architecture of the COVLIAS 1.0 (Figure 1) using HDL and SDL. The experimental protocol is shown in Section 3, while results and comprehensive performance evaluation are presented in Section 4 . The discussions and benchmarking are presented in Section 5. Finally, the paper concludes in Section 6. 


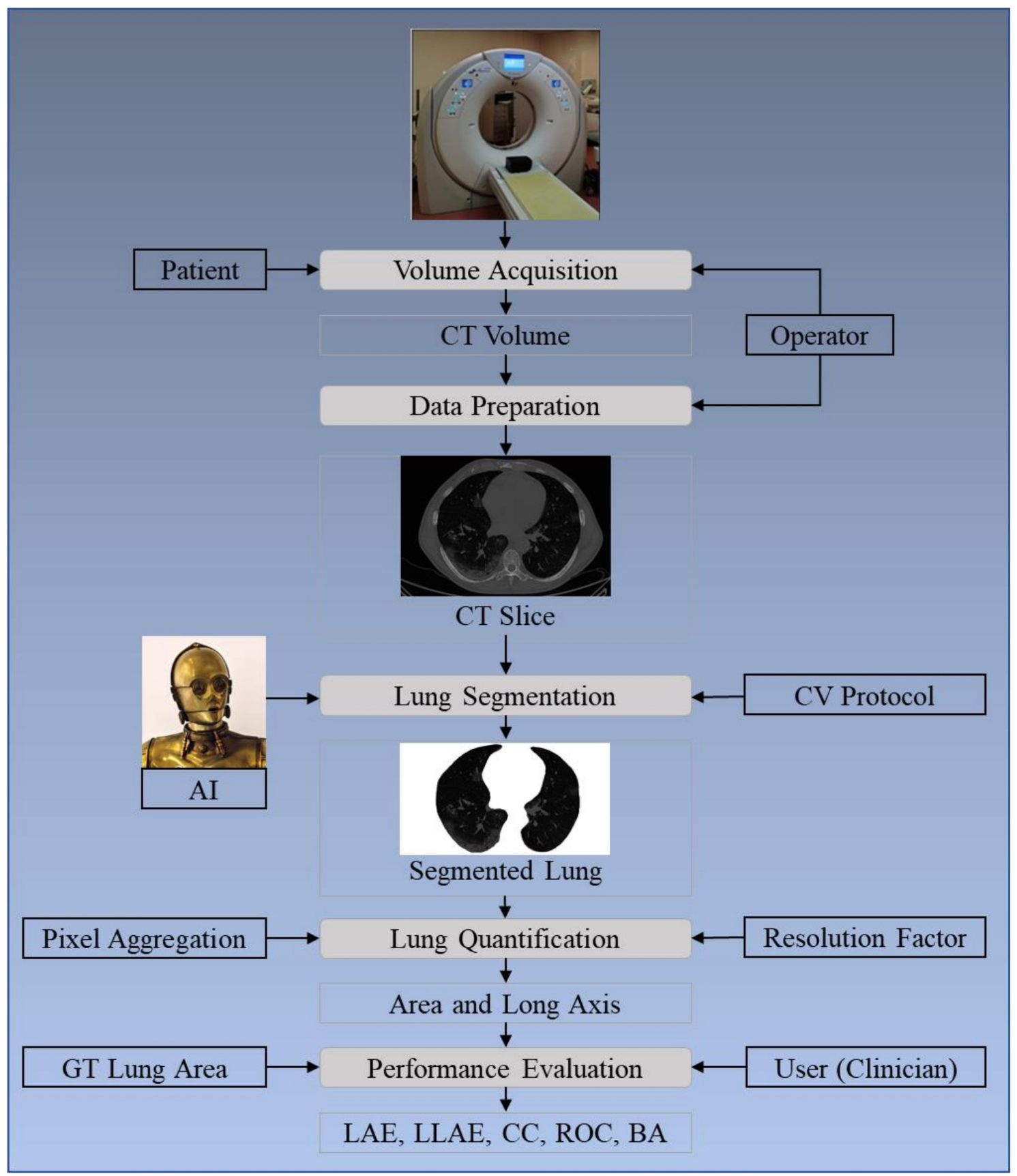

Figure 1. COVLIAS 1.0: Global system for lung segmentation and evaluation. AI: Artificial Intelligence; CV: CrossValidation; GT: Ground Truth; LAE: Lung Area Error; LLAE: Lung Long Axis Error; CC: Correlation coefficient; ROC: Receiver operating characteristic; BA: Bland-Altman.

\section{Methodology}

2.1. Patient Demographics, Image Acquisition, and Data Preparation

2.1.1. Demographics

The dataset consists of 72 Italian patients, where 46 patients were male and 26 were female. The mean height and weight were $172.72 \mathrm{~cm}$ and $79 \mathrm{~kg}$, respectively. A total of 60 patients were tested for PCR, while 12 could not be tested for PCR.

\subsubsection{Image Acquisition}

All chest computed tomography (CT) scans were performed during a single full inspiratory breath-hold in a supine position on a 128-slice multidetector-row CT scanner 
(Philips Ingenuity Core, Philips Healthcare, the Netherlands). No intravenous or oral contrast media were administered. The CT examinations were performed at $120 \mathrm{kV}$, $226 \mathrm{mAs}$ (using automatic tube current modulation-Z-DOM, Philips), with 1.08 spiral pitch factor, 0.5 -s gantry rotation time, and $64 * 0.625$ detector configurations. One-mm thick images were reconstructed with soft tissue kernel using $512 \times 512$ matrix (mediastinal window) and lung kernel using $768 \times 768$ matrix (lung window). CT images were reviewed on the Picture Archiving and Communication System (PACS) workstation equipped with two $35 \times 43 \mathrm{~cm}$ monitors produced by Eizo, with a $2048 \times 1536$ matrix. Figure 2 shows the raw sample CT scans of COVID-19 patients with varying lung sizes and variable intensity patterns, posing a challenge.

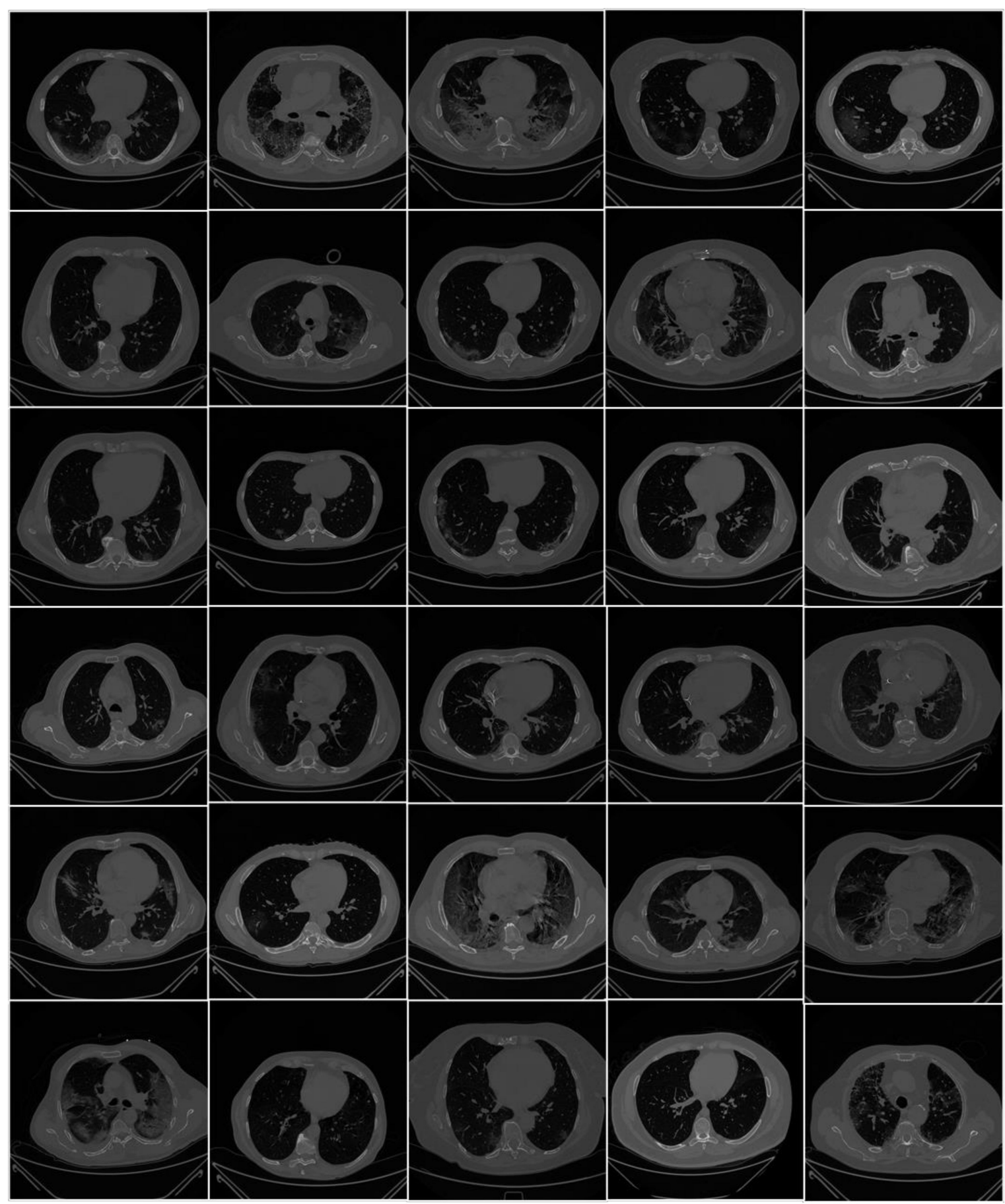

Figure 2. Raw lung COVID-19 CT images were taken from different patients in the database. 


\subsubsection{Data Preparation}

The CT data of 72 COVID-positive patients' data are used in the proposed study. Each patient consisted of 200 slices, out of which 65-70 slices consisting of visible lung region were selected by the radiologist [LS]. A total of 5000 images were used in the proposed study. A total of 2000 images were used to train the AI-based segmentation models, whereas 3000 images were used to test and validate the models.

To prepare the data for segmentation, a binary mask was generated manually by tracing in the selected slices under the guidance of a trained radiologist [LS] using ImgTracer ${ }^{\mathrm{TM}}$ (Global Biomedical Technologies, Inc., Roseville, CA, USA) [38-40]. Figure 3 shows the raw lung COVID-19 CT scans with ground truth boundary (white) overlay using manual tracing. Figure 4 shows the white binary mask of the lung region computed using manual ImgTracer ${ }^{\mathrm{TM}}$.

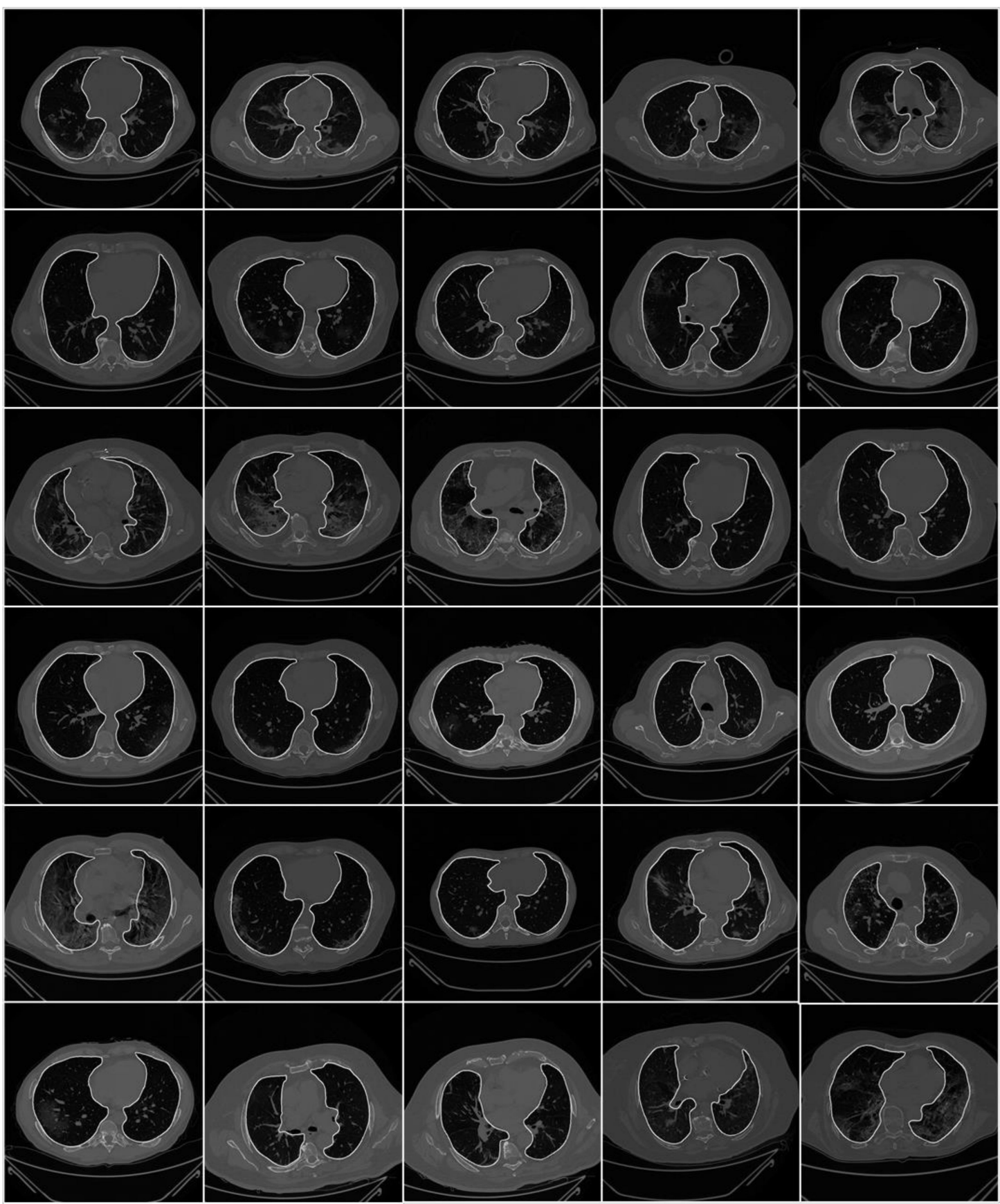

Figure 3. Raw lung images with ground truth boundary (white) overlay on grayscale CT scans. 


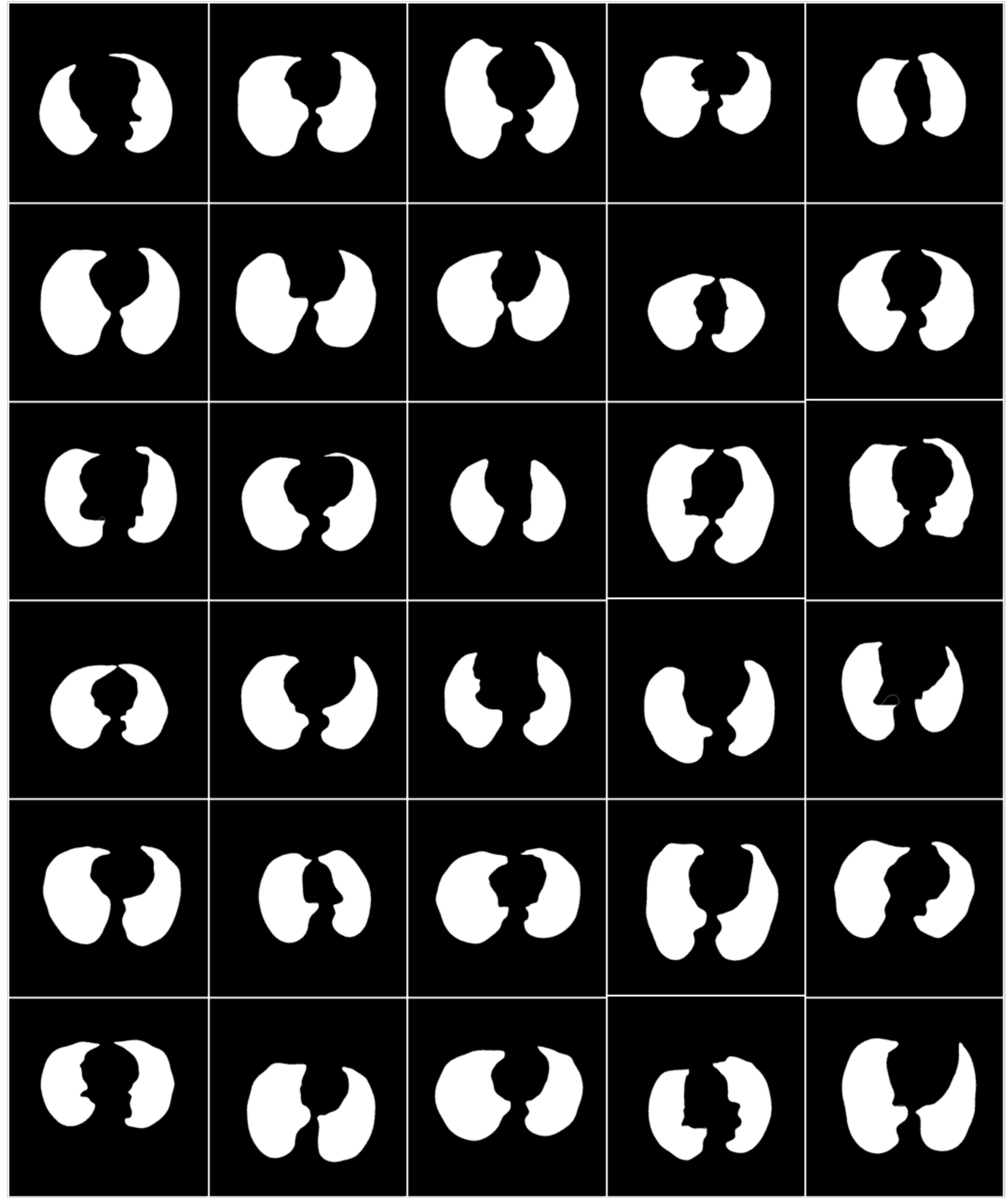

Figure 4. White binary mask of lung region used for AI-based model training.

\subsection{Architecture}

The COVLIAS 1.0 system incorporates three models: deep learning-based (SDL) and two hybrid-based (HDL). We benchmark these models against the conventional NIHbased model using fuzzy connectedness. In all, the proposed study incorporates four 
kinds of models such as (a) SegNet, (b) VGG-SegNet, (c) ResNet-SegNet, and (d) NIHconventional model.

\subsubsection{SegNet}

SegNet is a semantic segmentation model shown in Figure 5 and has been implemented by Badrinarayanan et al. before [41], while we adapted SegNet for comparison with HDL. SegNet is a trainable segmentation architecture that consists of (i) an encoder network (the left half of Figure 5), (ii) a corresponding decoder network followed by (the right half of Figure 5) (iii) a pixel-wise classification layer (the last block of Figure 5). The architecture of the encoder network is topologically identical to the 13 convolutional layers. It uses a technique to down-sample encoder output, which involves storing the max-pooling (filter size of $2 \times 2$ ) indices. The max-pooling layer is placed at the end of each block on the encoder side, which increases the depth of the following layer by two times (64-128-256-512).

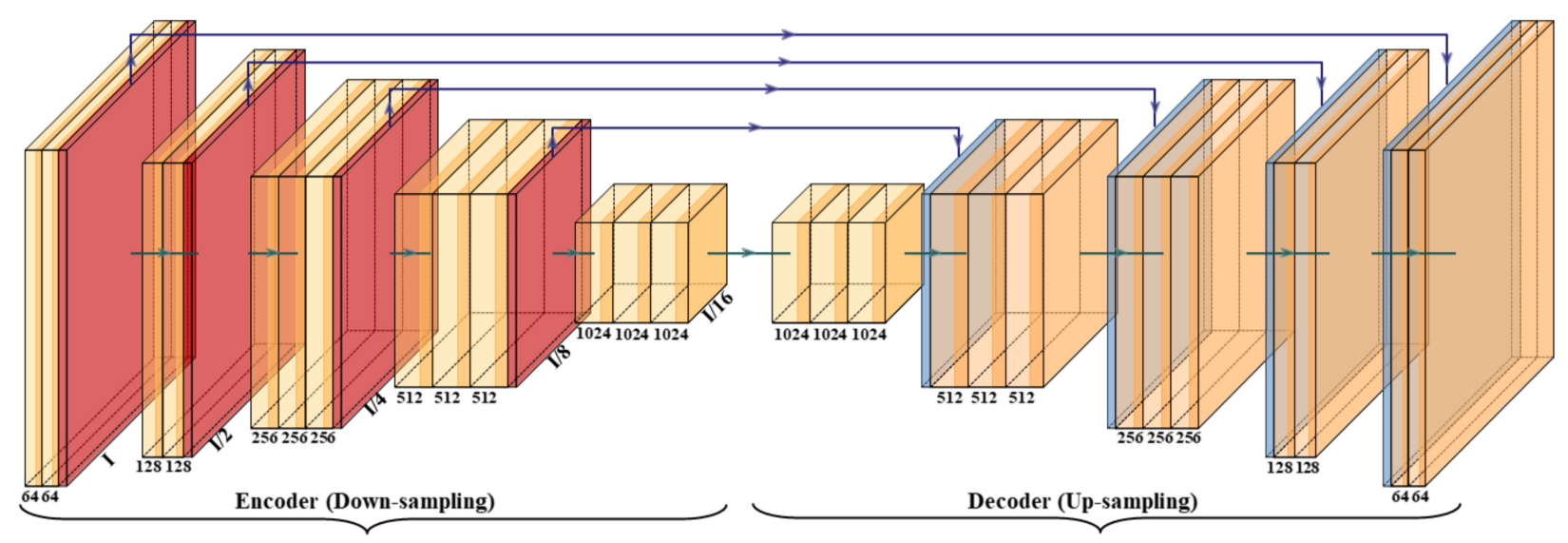

Figure 5. SegNet architecture.

Similarly, in the second half of the architecture, the up-sampling happens where the depth of the layer decreases by two (512-256-128-64). The up-sampling process involves recalling the max-pooling indices at the corresponding encoder layer. Finally, in the end, there is a K-class softmax classifier used to predict the class for each pixel. This gives reasonably good performance and is space-efficient.

\subsubsection{VGG-SegNet}

The VGG architecture consists of blocks, where each block is composed of 2D convolution (Conv) and max-pooling layers (Figure 6). VGGNet was born out of the need to reduce the \# of parameters in the convolution layers and improve training time [42]. This architecture consists of 16 convolution layers compared to 13 in SegNet. It helps the model to learn high-level features to improve the results. The blocks used in the VGG-SegNet architecture consist of (i) encoder, (ii) decoder part, and followed by (iii) a pixel-wise softmax classifier.

The distribution of the blocks in the encoder (the left half of Figure 6$)$ is $2 \times(2$ Conv + max-pool $)+3 \times(4$ Conv + max-pool), and the decoder (the right half of Figure 6$)$ is the reverse of the encoder part with up-sampling, thereby decreasing the depth of the layers. 


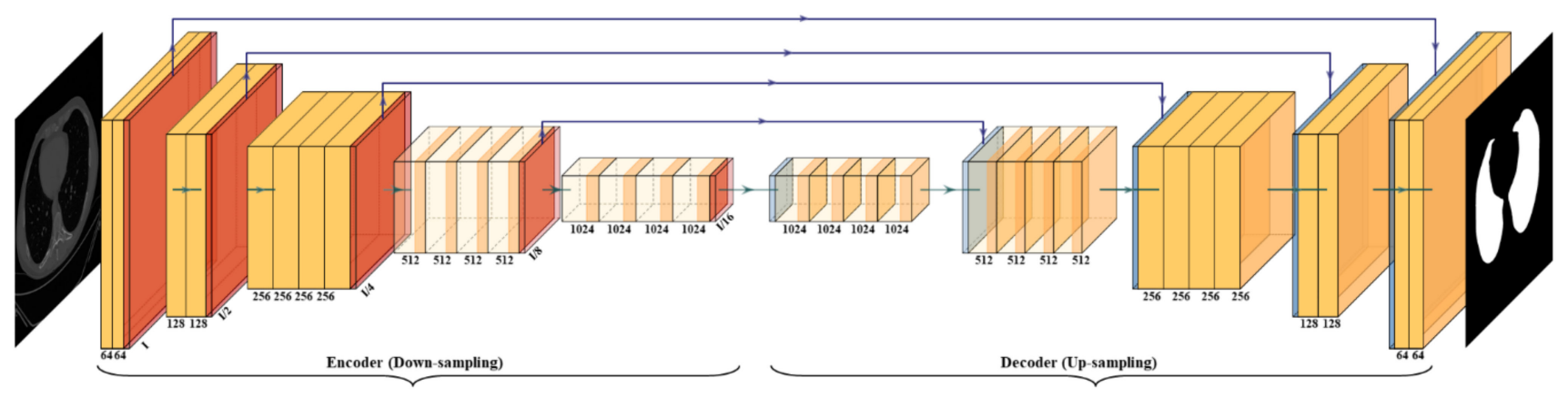

Figure 6. VGG-SegNet architecture.

\subsubsection{ResNet-SegNet}

Residual Network (ResNet) [43] came into existence by overcoming the VGG architecture problem of the vanishing gradient. When the network goes through backpropagation, the gradient keeps flowing backward to the initial layers. This value keeps getting multiplied by each gradient. As a result, the gradient becomes smaller and smaller, making the updates to the initial layers very small, also known as the vanishing gradient problem. Consequently, this results in an increase of training time. ResNet is a superior architecture to VGG because it has skip connections, which act as gradient superhighways or bypasses, allowing the gradient to flow unhindered. This solves the problem of diminishing gradient. With the help of the identity function (shown in the bottom right of Figure 7), the value of backpropagated gradient values does not decrease because the local gradient value is one.

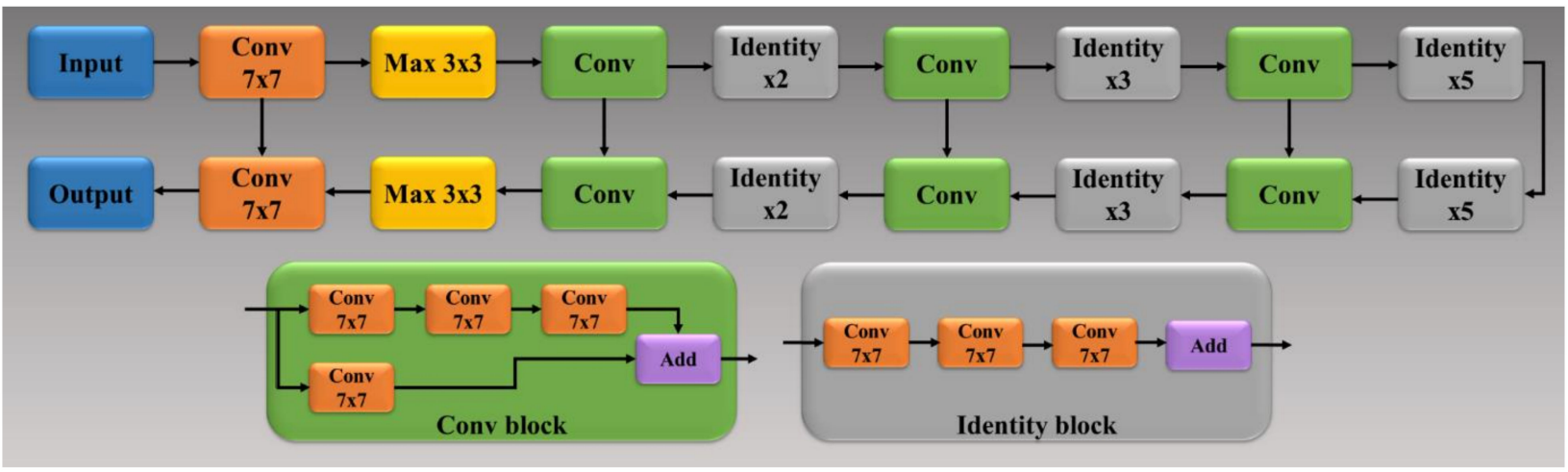

Figure 7. ResNet-SegNet architecture.

\subsubsection{NIH Segmentation Model}

Figure 8 shows the architecture of the fuzzy-connected system for segmentation of the lung region. This system was not built in the study but simply adapted from the NIH website [37] for benchmarking. There are three steps in the process: (i) seed point selection, (ii) fuzzy-connected (FC) segmentation and binary mask cleaning, and (iii) grayscale segmentation. The user can select the seed point in two lines corresponding to the left and right lungs. FC segmentation is executed, and the results are displayed in the form of a binary mask. Finally, the lung is segmented with the given binary mask and the original CT COVID-19 grayscale scan leading to segmented lungs. 


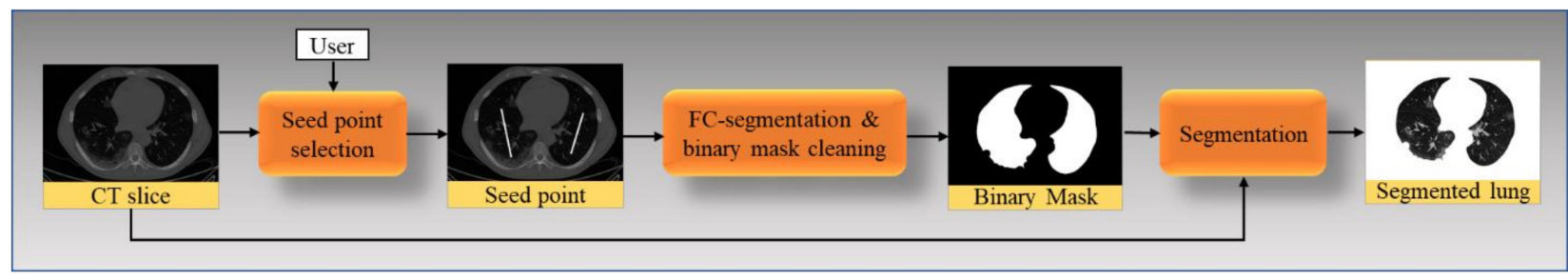

Figure 8. User interactive fuzzy-connectedness Center for Infectious Disease Imaging (CIDI) lung segmentation [37].

\subsubsection{Loss Functions for SDL and HDL Models}

The proposed system uses CE-loss in SDL/HDL models. The CE-loss function, labeled as $L_{C E}$ for all the models, can be mathematically given by Equation (1):

$$
L_{C E}=-\left[\left(\mathrm{y}_{\mathrm{i}} \times \log \mathrm{a}_{\mathrm{i}}\right)+\left(1-\mathrm{y}_{\mathrm{i}}\right) \times \log \left(1-\mathrm{a}_{\mathrm{i}}\right)\right]
$$

where $y_{i}$ is the input GT label $1,\left(1-y_{i}\right)$ is GT label $0, a_{i}$ represents the softmax classifier probability, and $x$ represents the product of the two terms. All three of the AI architectures shown in Figures 5-7 have been trained using the CE-loss function.

\subsection{Sample Size Calculation}

It is vital to understand the sample size needed for achieving the desired performance. Generally, sample size requires calculating the number of studies adapted in our proposed system for reaching the desired accuracy and error statistics. This is crucial because, when the sample size is too large with respect to the desired sample size, it may incur more computational and economic burdens both in terms of time and cost. We follow paradigms like our previous paradigms for sample size calculation [27,44-49]. To represent the mathematical formula for the sample size calculation, we used a z-score from the standard z-table as shown by Equation (2):

$$
N_{p}=(z)^{2} \times \frac{\hat{p}(1-\hat{p})}{(\mathrm{MoE})^{2}}
$$

In the proposed study, we use a confidence level of $95 \%$, a margin of error (MoE) of $5 \%$, and a data proportion $(\hat{p})$ of 0.5 . Normal distribution was assumed for the data sample, and a confidence interval of $95 \%$ provided a $z$-score value of 1.96 (derived from the standard z-table). MoE of 5\% for SDL and 3\% for HDL was used to ensure that the population remains in the tolerance band. The desired sample size $(n)$ for a large, true population size was computed using Equation (2). Using the confidence level of $95 \%$, the resultant sample size resulted in 1351 samples while using MoE of 3\% for HDL and 588 samples for SDL with 5\% MoE. Thus, the recruited sample size for HDL and SDL was $\sim 270 \%$ and $\sim 750 \%$ higher than the desired sample size.

\section{Experimental Protocol}

\subsection{Cross-Validation}

A total of 5000 COVID-19 CT slices are used in the proposed system. A well-known data partitioning method, K5-r46, was used in our system, in which the database was partitioned into five parts (K5). Then, a 4:6 ratio was used for training to test, these $40 \%$ of (2000 images) data are used for training, and 60\% (3000 images) of data are used for testing. An internal validation system was also used in our system with training data. For each test patient, the binary mask was generated, and the lung area was estimated.

\subsection{Lung Quantification}

The lung area is measured by counting the number of white pixels in the lung region and converted to $\mathrm{mm}^{2}$ dimensions using the resolution factor of $0.05 \mathrm{~mm}$ to a pixel. If $N$ represents the total number of the image in the database, $\mathcal{A}_{a i}(m, n)$ represents lung 
area for in the image " $n$ " using the AI model " $m$ ", $\overline{\mathcal{A}}_{a i}(m)$ represents the mean lung area corresponding to the AI model " $m$ ", $\overline{\mathcal{A}}_{g t}$ represents the corresponding mean area of the GT binary lung mask, then mathematically $\overline{\mathcal{A}}_{a i}(m)$ and $\overline{\mathcal{A}}_{g t}$ can be computed and are shown in Equation (3):

$$
\left.\begin{array}{rl}
\overline{\mathcal{A}}_{a i}(m) & =\frac{\sum_{n=1}^{N} \mathcal{A}_{a i}(m, n)}{N} \\
\overline{\mathcal{A}}_{g t} & =\frac{\sum_{n=1}^{N} \mathcal{A}_{g t}(n)}{N}
\end{array}\right\}
$$

\subsection{Accuracy Computation}

The accuracy of the AI system is measured by comparing the predicted output and the ground truth pixel values. These values were interpreted as binary ( 0 or 1$)$ numbers as the output lung mask was only black and white, which was then summed up and divided by the total number of pixels. Assuming TP represents true positive, TN represents true negative, FN represents false negative, and FP represents false positive. The accuracy of the AI system can be given as shown in Equation (4) [50]:

$$
\operatorname{ACC}(\%)=\left(\frac{\mathrm{TP}+\mathrm{TN}}{\mathrm{TP}+\mathrm{FN}+\mathrm{TN}+\mathrm{FP}}\right) \times 100
$$

\section{Results and Performance Evaluation}

\subsection{Results}

COVLIAS 1.0 was designed to run $40 \%$ training and $60 \%$ unseen data sets from 5000 scans. The output of the system reported the binary mask for the left and right lung jointly. These masks were then used for computing the segmented region, given the CT grayscale COVID-19 scans. Figure 9 shows the segmented mask (column 2), segmented lung region (column 3), and overlay image of segmented lung mask of green color over the grayscale image (column 4). Figure 10 shows the accuracy (Equation (4)) and the loss functions (Equation (1)) using the three AI models such as SegNet, VGG-SegNet, and ResNet-SegNet. The accuracy and loss values were then plotted as a stacked line chart to show the increase in the accuracy and decrease in the loss of the training model.

\subsection{Performance Evaluation}

As part of the performance evaluation, three metrics and nine evaluation criteria were considered. First are the visualization comparisons, second is the lung area metrics, and third are the long axis metrics. Section 4.2.1 presents the visualization of boundary error and lung area error (LAE). It has two sets of visualization components (i) visualization of the model boundary against the ground truth boundary with different boundary colors; and (ii) visualization of the superimposition of the model lung region against the GT lung region with different colors; Section 4.2.2 presents the performance evaluation based on the LAE and consists of (i) cumulative plot of the LAE; (ii) Bland-Altman plot for the LAE; (iii) JI and DS for the lung region and (iv) ROC curves for AI-based model performance. Section 4.2.3 presents the performance evaluation based on the lung long axis error (LLAE) and consists of the following components: (i) cumulative plot of the LLAE; (ii) correlation coefficient of the LLAE; and (iii) Bland-Altman plot for the LLAE.

The lung masks were then converted into lung boundary images using a region-toboundary convertor. The lung boundary was superimposed over the original COVID-19 lung CT grayscale scans to visualize the lung, shown in Figure 11. Note that the top three AI models (1 SDL and 2 HDL) performed well (row-1, row-2, and row-3), as can be seen visually, unlike the NIH conventional model (row-4). 


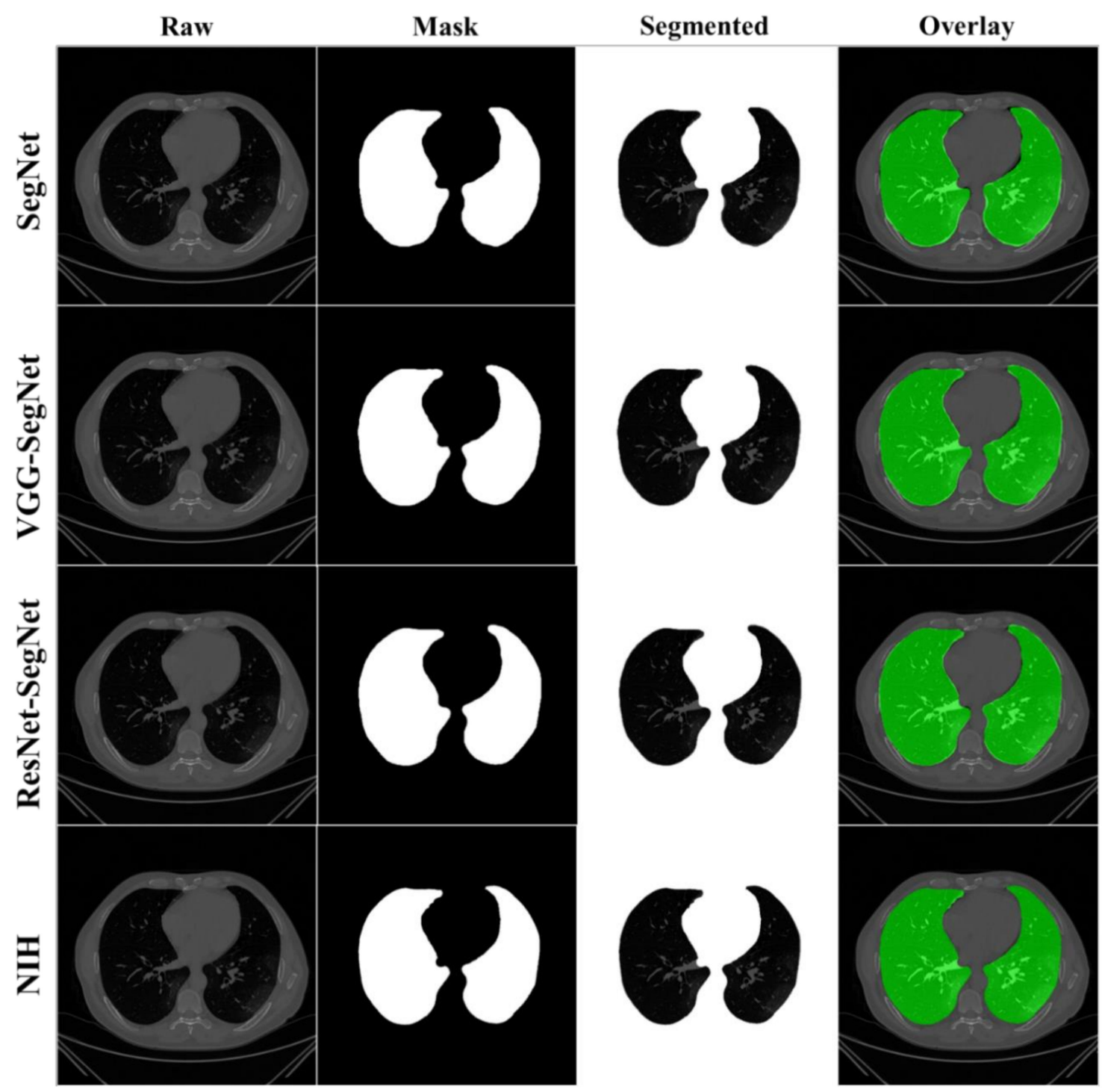

Figure 9. Results from the four AI models with grayscale CT slice. Row 1 to Row 4 is four models: SegNet, VGG-SegNet, ResNet-SegNet, and NIH. 

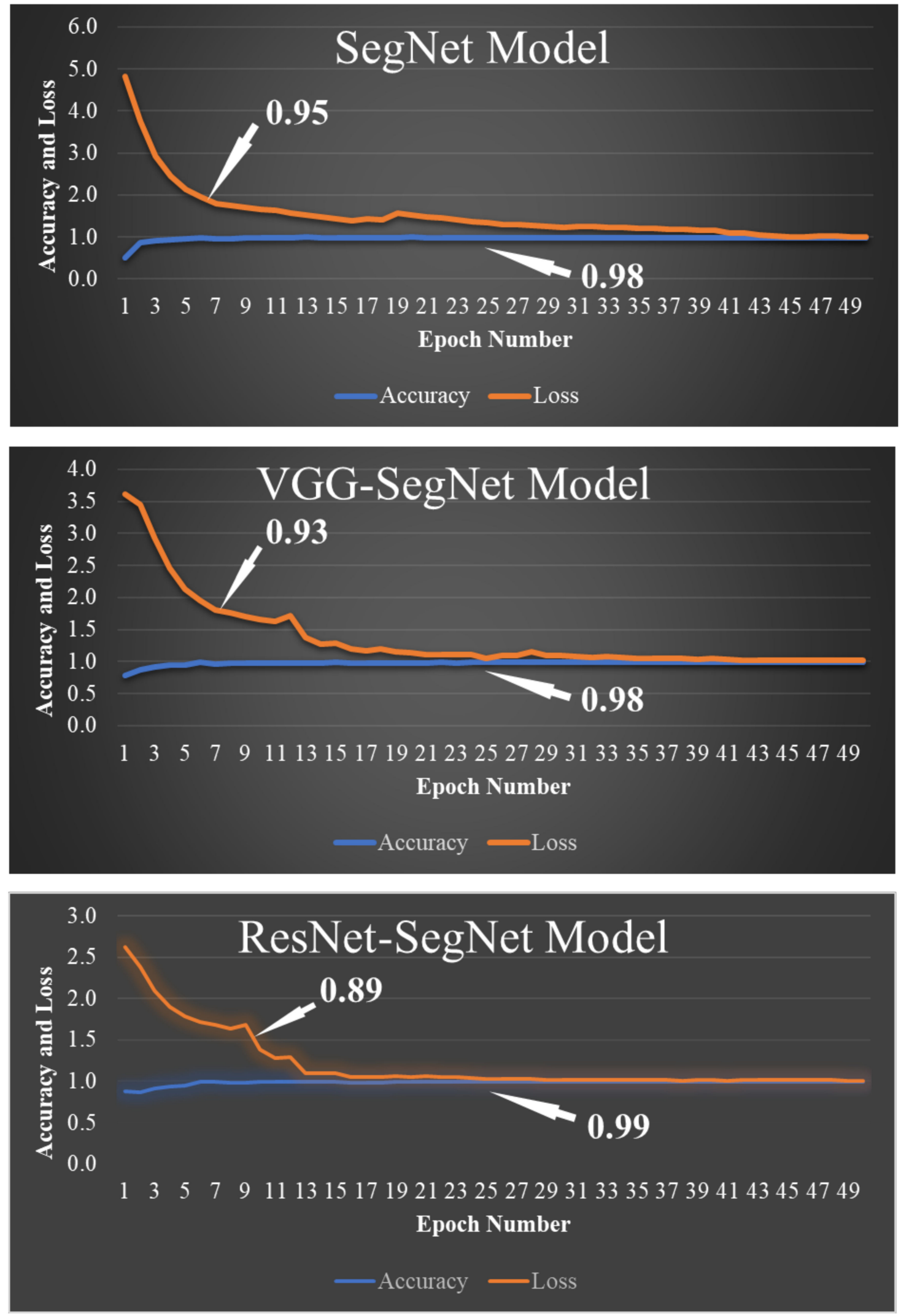

Figure 10. Accuracy and loss from the three AI models SegNet, VGG-SegNet, and ResNet-SegNet. 


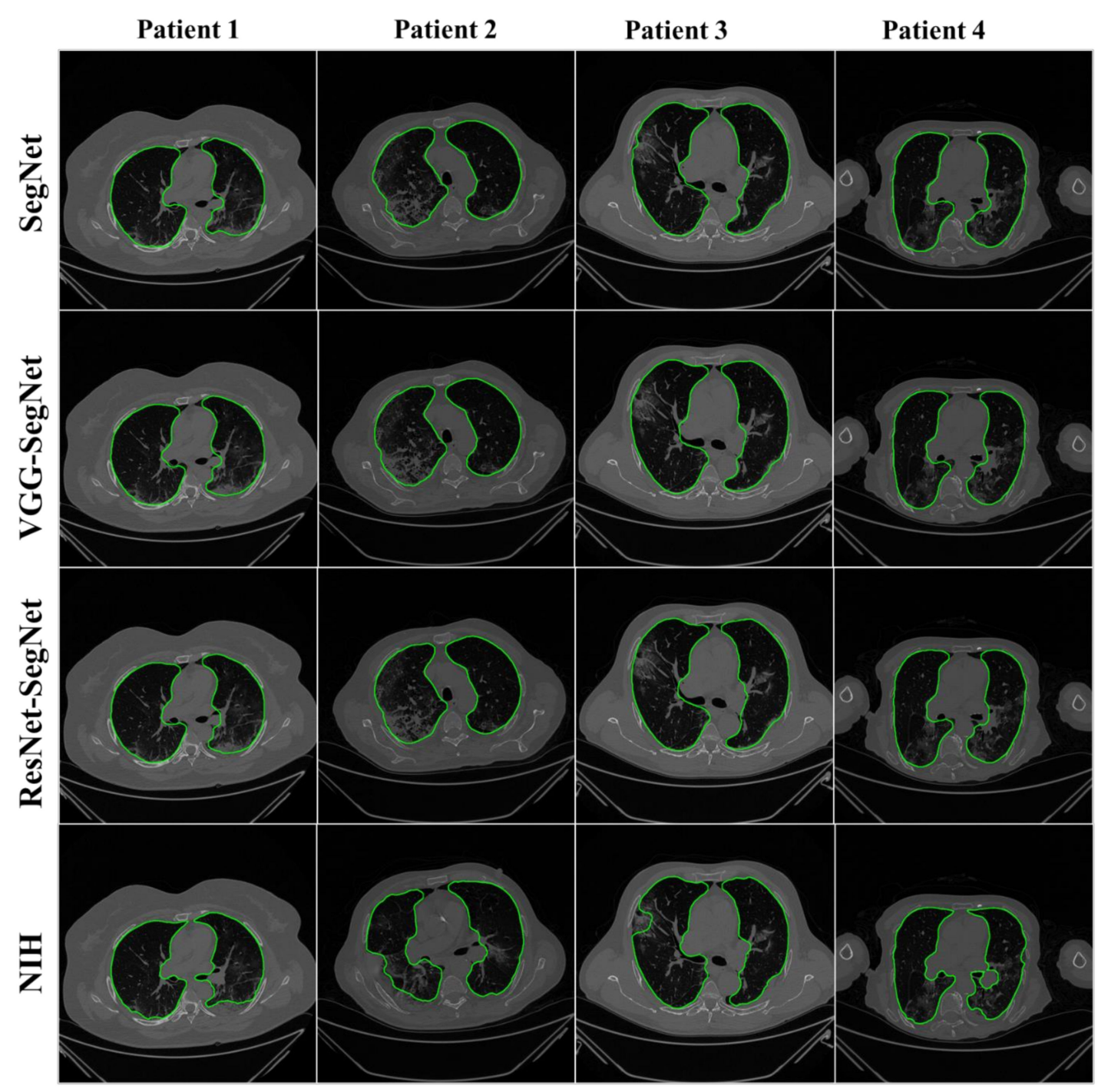

Figure 11. Results from the four AI models and NIH with a grayscale CT slice. Row 1 to Row 4 is four models: SegNet, VGG-SegNet, ResNet-SegNet, and NIH.

\subsubsection{Visualization of Lung Boundary and Regional Lung Error}

Figure 12 shows the overlay of the AI-model boundary (green) and GT-boundary (red) with a grayscale COVID-19 CT slice in the background. Four models are shown in the rows in the following order: SegNet, VGG-SegNet, ResNet-SegNet, and NIH. As we go down the rows, the boundaries are bumper-to-bumper and show the nearly negligible difference between three AI models and GT, while the pronounced error for the NIH model. The white arrows indicate the region of mismatch between the NIH model and ground truth. The top three rows (SegNet, VGG-SegNet, and ResNet-SegNet) show more closeness between the AI-model borders (green) and the ground truth borders (red). 


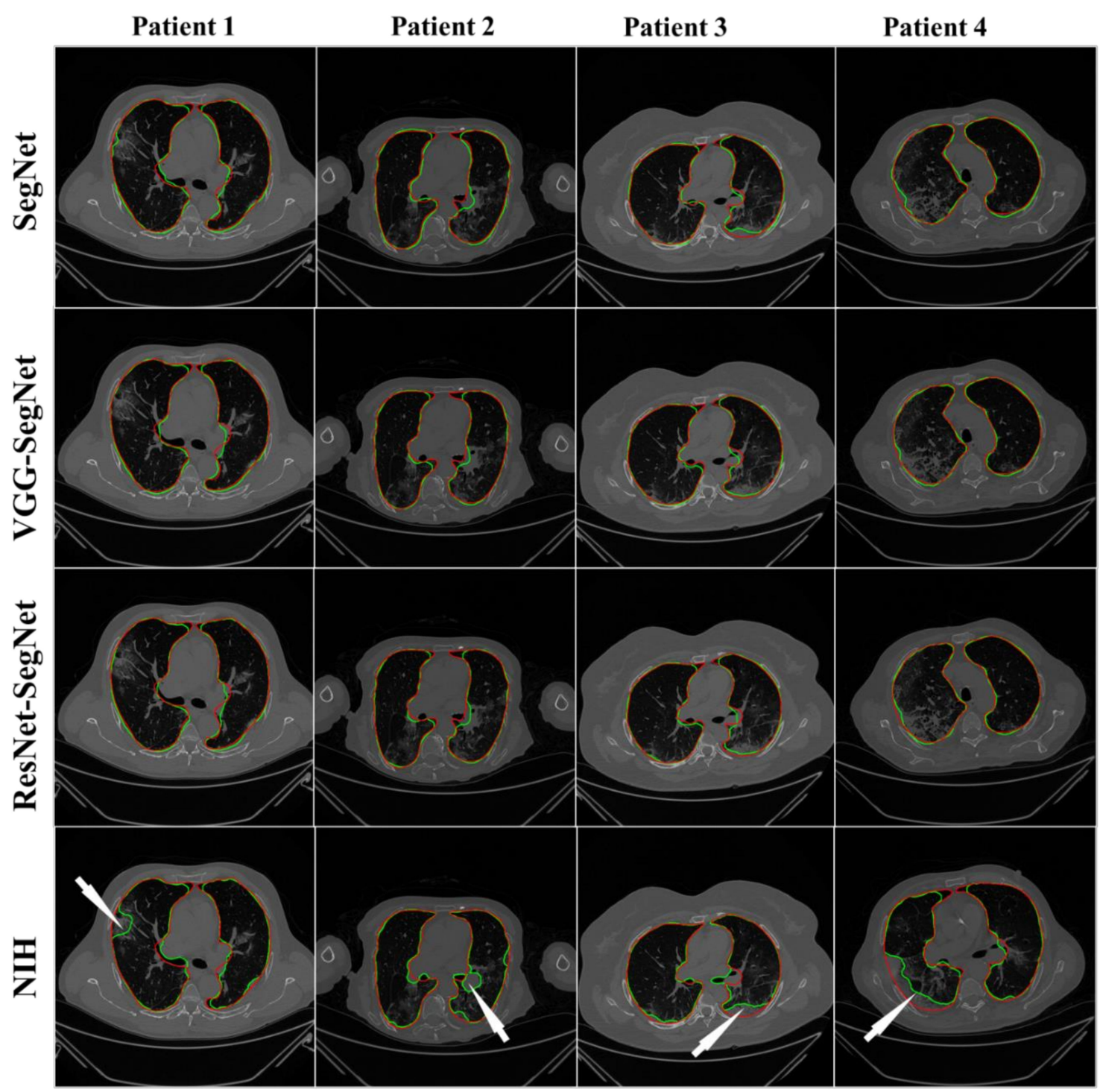

Figure 12. Boundary overlay of the segmented mask from the AI-model (green) vs. GT-mask in the background (red). White arrow shows NIH-segmented boundary not at a right position.

Figure 13 shows the lung region using AI-mask from the AI-model (green) vs. GTmask (red). Note that the slight difference is visible between the two-colored masks for the first three AI models, while a more significant difference is seen between the NIH model and GT, typically at the basal region (shown by the white arrows). For better visualization of the differences in area region, we have taken two sample slices in Figure 14, where the combination (c) shows the zoomed version of (a), and combination (d) shows the zoomed version of (b). Figure 15 shows the long axis of the AI-model (green) and GT (red) with a grayscale COVID-19 CT slice in the background. Four models are shown in the rows in the following order: SegNet, VGG-SegNet, ResNet-SegNet, and NIH. 


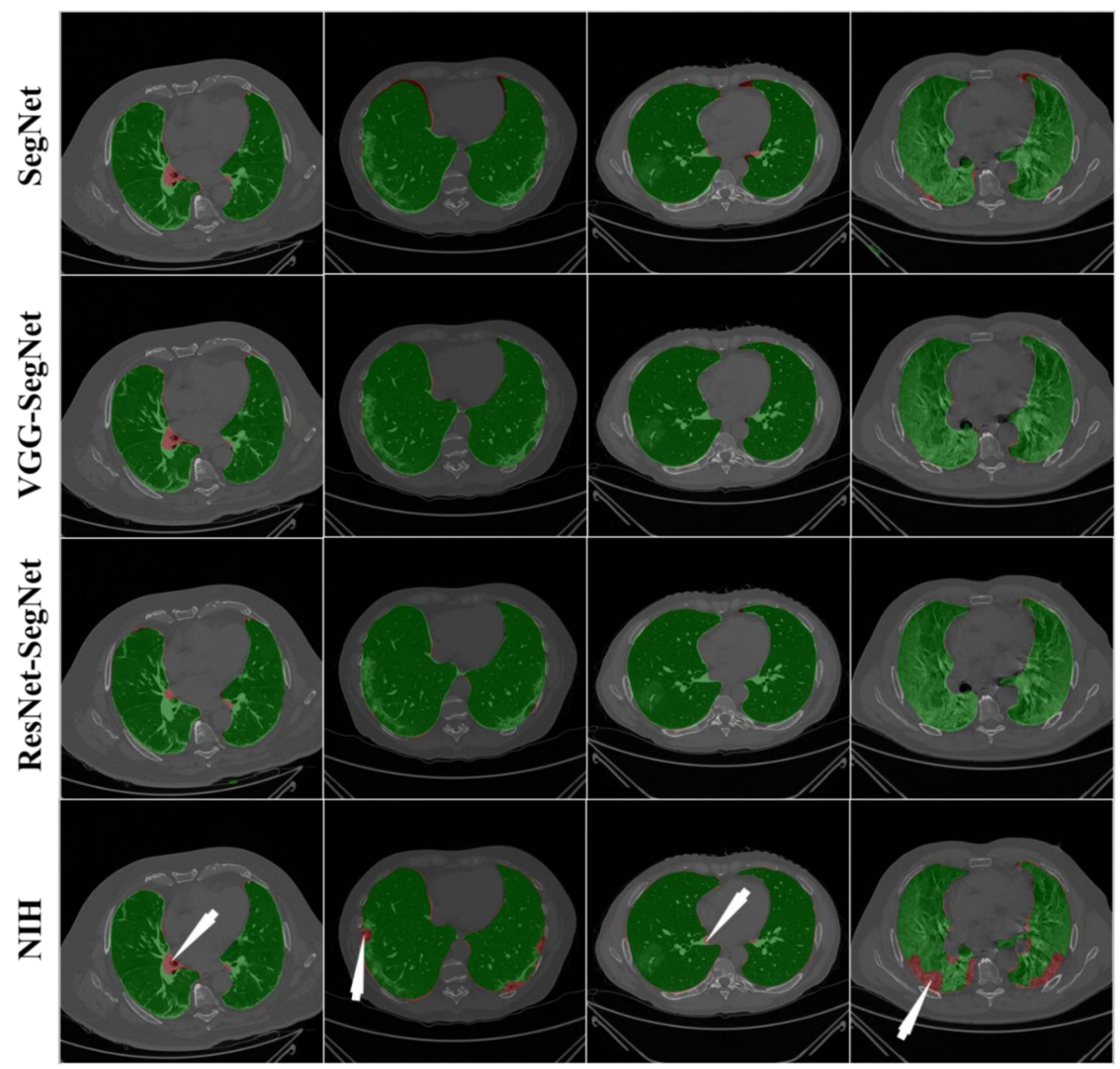

Figure 13. Overlay of the segmented mask from the AI-model (green) vs. GT-mask in the background (red). White arrow shows NIH-segmented boundary not at a right position. 


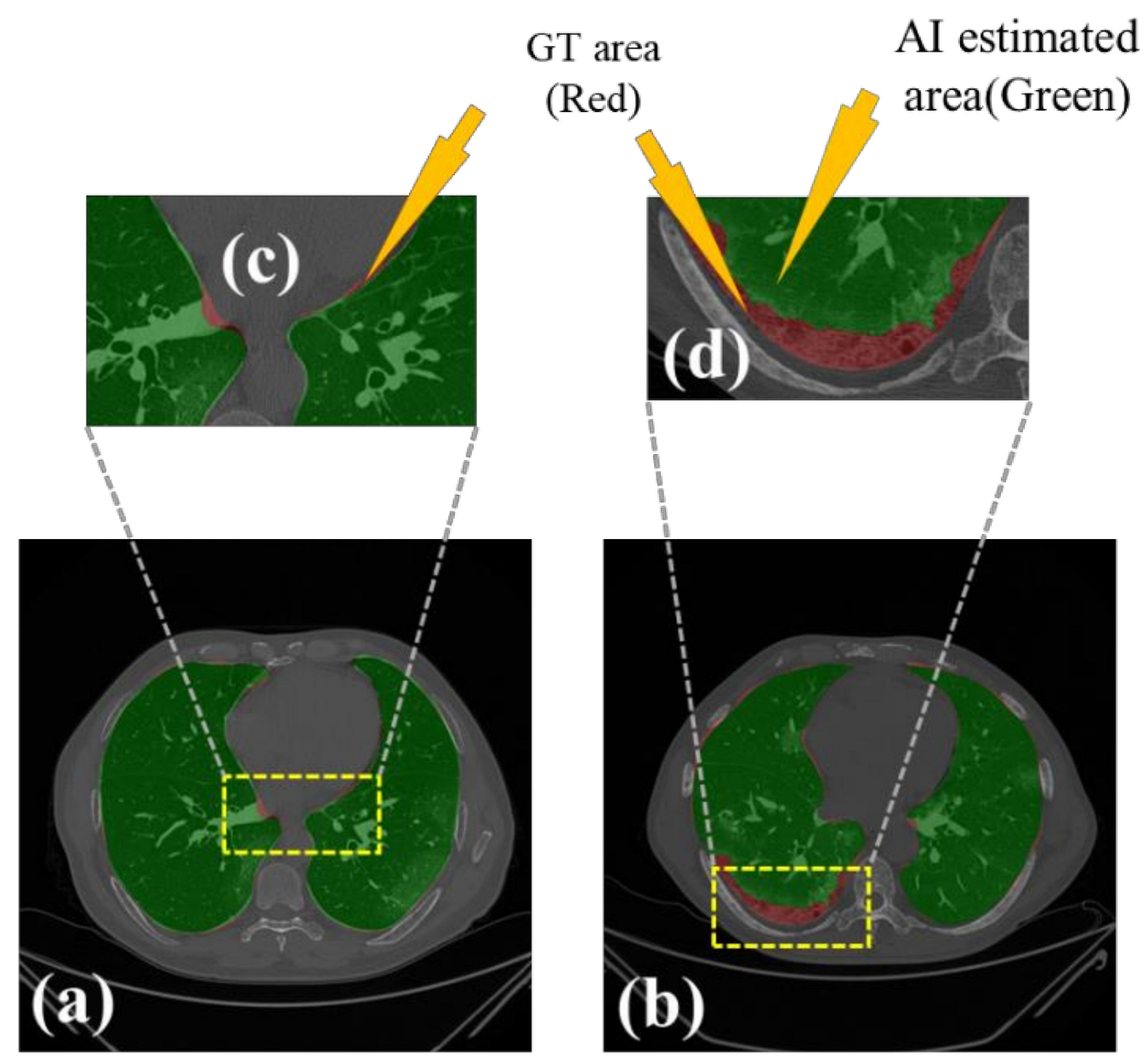

Figure 14. Visualization of the zoomed region-of-interest showing the difference between AI area (green) and GT area (red). (a) central lung region, (b) basal region of the lung, (c) zoom of the central region, and (d) zoom of the basal region. Arrow shows the difference between GT and AI area. 


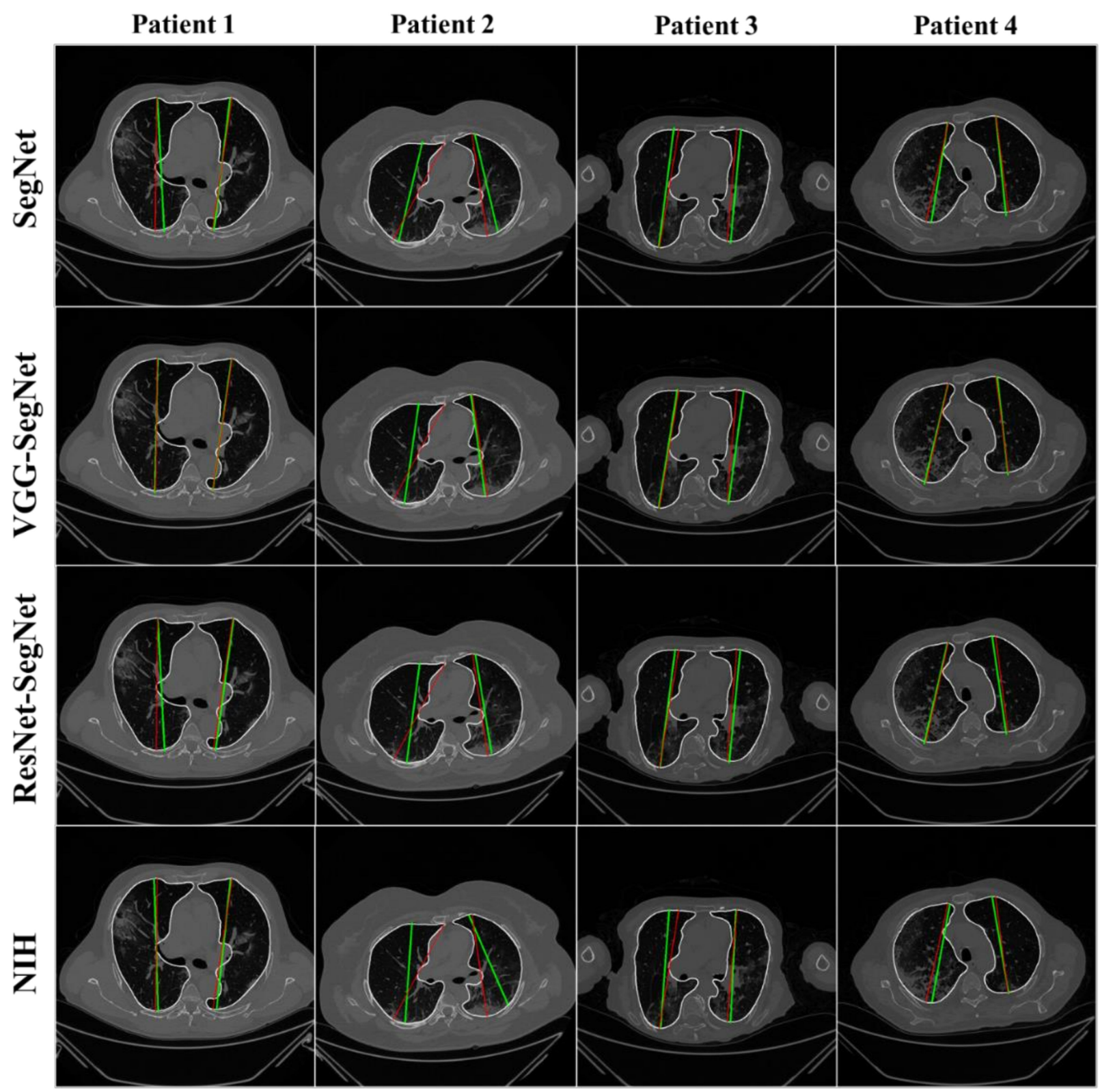

Figure 15. Display of the AI-long axis (green) and GT-long axis (red) showing the reach of the top apical zone and bottom basal zone of the left and right lungs. Rows are the four models, and columns are sample four patients COVID-19 CT scans. White is the GT delineated borders of the lungs by the tracer using ImgTracer(TM).

\subsubsection{Performance Metrics for the Lung Area Error}

Cumulative Plot for Lung Area Error

Cumulative frequency analysis is the analysis of the frequency of occurrence of the LAE against the reference value. Figure 16 below shows the cumulative distribution of left and right LAE over 3000 images corresponding to the test dataset (60\% of 5000 scans). A cumulative frequency threshold of $80 \%$ was chosen to compare the four different models: SegNet, VGG-SegNet, ResNet-SegNet, and NIH. Note that, even though the AI models perform exceptionally well, the best of all was the HDL model ResNet-SegNet with 80\% LAE for left, right, and combined left/right as $8.3 \mathrm{~mm}^{2}, 5.8 \mathrm{~mm}^{2}$, and $7.5 \mathrm{~mm}^{2}$, respectively. Furthermore, note that the NIH showed the lowest performance at an $80 \%$ threshold $\left(19.83 \mathrm{~mm}^{2}, 13.12 \mathrm{~mm}^{2}\right.$, and $19.22 \mathrm{~mm}^{2}$, corresponding to the left lung, right lung, and mean of the left lung and right lung). It is also interesting to note that the right LAE was lower than the left LAE for all four models. 

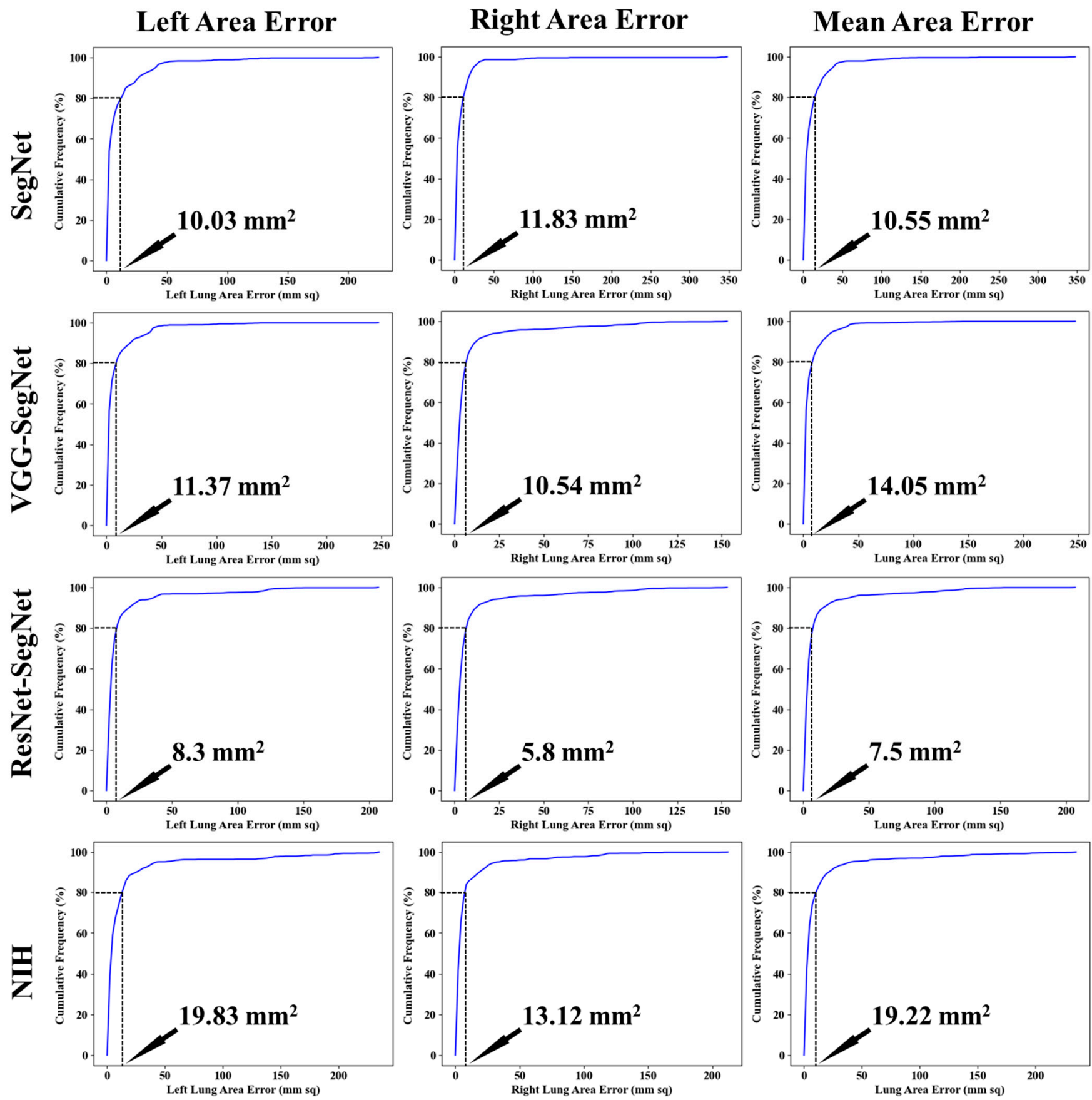

Figure 16. Cumulative plot for LAE for the four models corresponding to left, right, and mean.

\section{Lung Area Error}

Figure 17 shows the increasing LAE between the AI-models and GT for the left, right, and mean areas was in the order of ResNet-SegNet $<$ VGG-SegNet $<$ SegNet $<$ NIH. This is consistent with Figure 16, where the LAE increases in the order of ResNet-SegNet $<$ VGG-SegNet $<$ SegNet $<$ NIH, demonstrating that AI models (SDL and HDL models) are superior to NIH conventional models. 


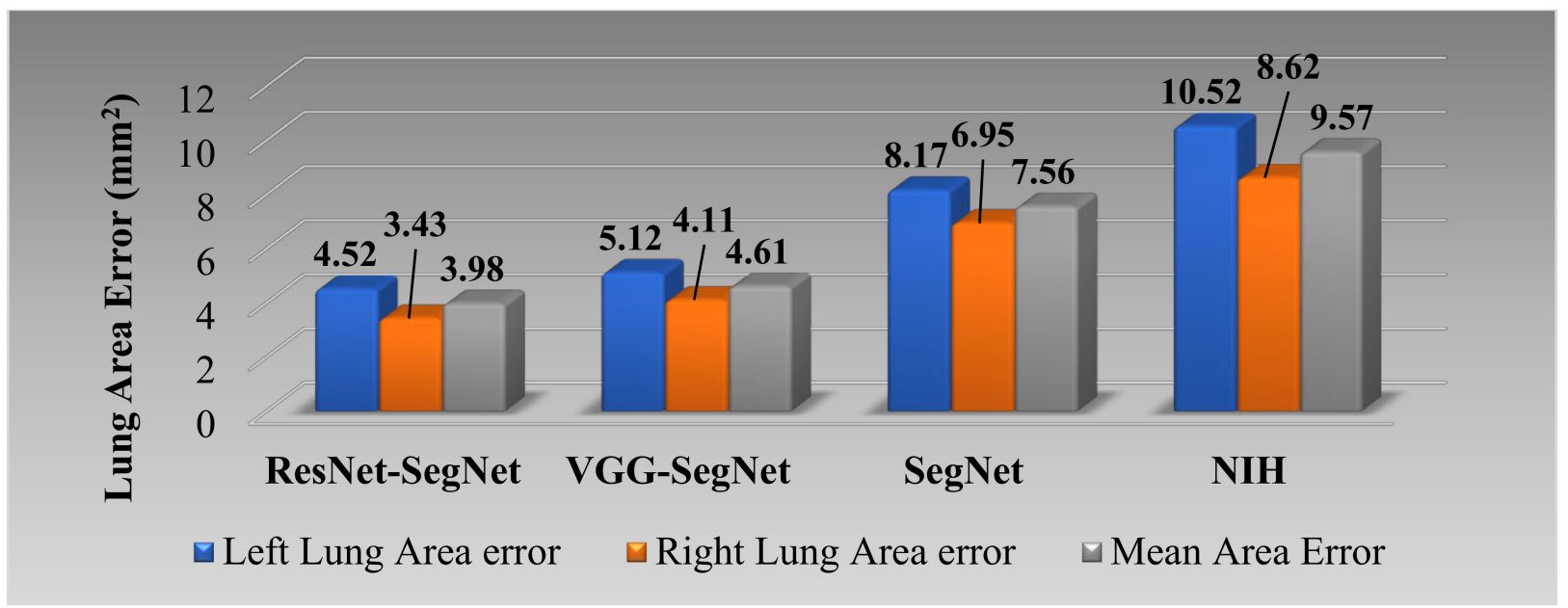

Figure 17. Bar Chart showing the LAE for the four models corresponding to left (blue), right (red), and mean (green) areas. LAE was in the following order ResNet-SegNet $<$ VGG-SegNet $<$ SegNet $<$ NIH.

\section{Correlation Plots for Lung Area}

Figure 18 ( $4 \times 3$ matrix) shows the coefficient of correlation (CC) plot for AI-model lung area vs. GT area using the four models (rows) corresponding to left, right, and mean (columns). The CC values of all the models ranged from 0.91 to 0.98 ( $p$-value $<0.0001$ ). The 3D bar plot is shown in Figure 19 for the left, right, and mean lungs. For the left lung, the decreasing order of the CC performance was ResNet-SegNet, VGG-SegNet, SegNet, and NIH. Thus, HDL models were better than the SDL model. This order remained the same for the left lung, right lung, and mean lung area. These results were consistent with Figures 16-18.
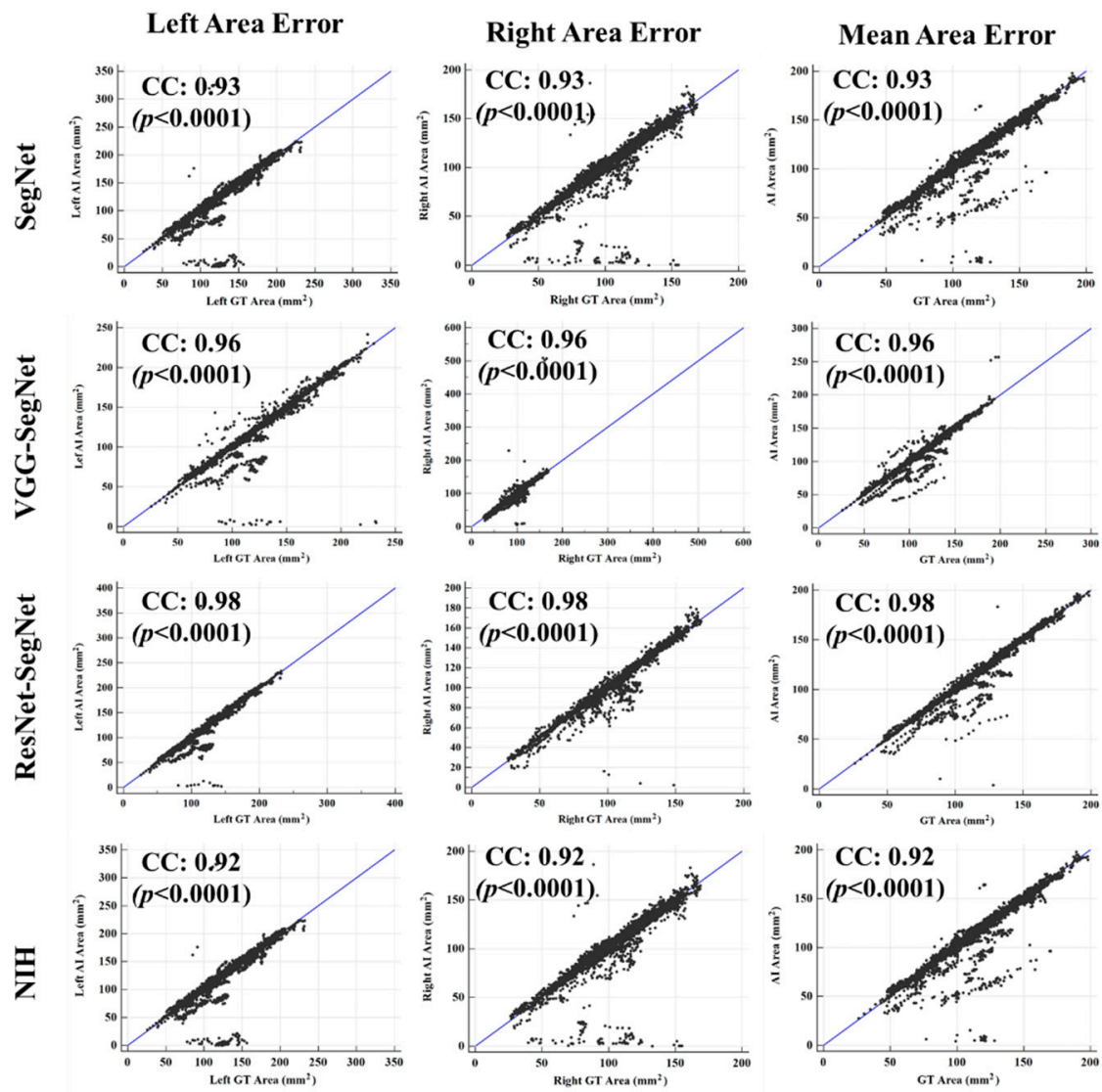

Figure 18. Correlation plot for lung area for four models corresponding to left, right, and mean. 


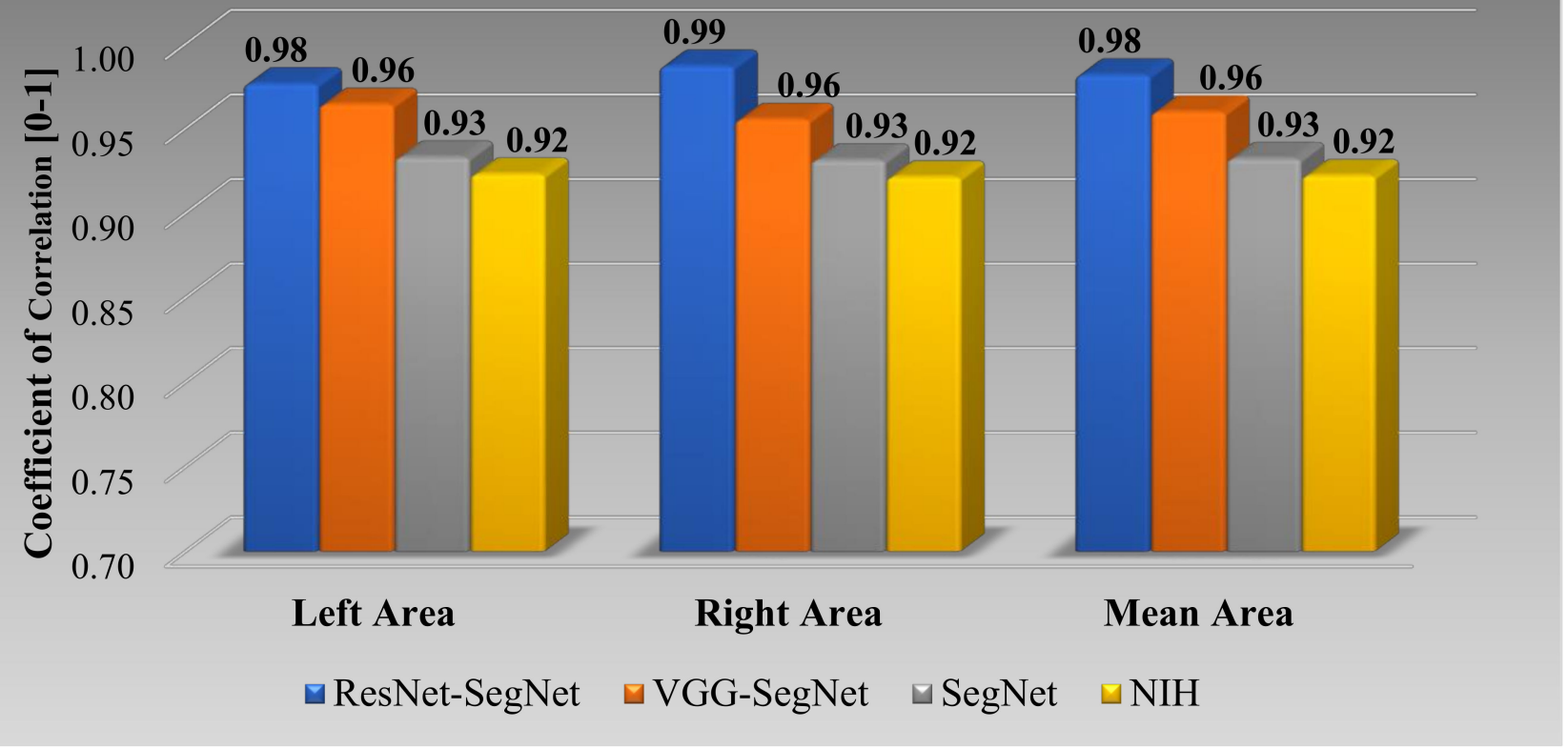

Figure 19. Plot for correlation coefficient between the AI model and GT area for the four types of model: ResNet-SegNet, VGG-SegNet, SegNet, and NIH for left lung, right lung, mean of the two lungs.

Jaccard Index and Dice Similarity for Lung Area

Figure 20 below shows the bar chart for the four models using the Jaccard index (JI) (blue) and Dice similarity (DS) (red). The performance of the models (best to worst) had the following order ResNet-SegNet $>$ VGG-SegNet $>$ SegNet $>$ NIH, consistent with Figures 16-19. The cumulative frequency for JI and DS for the four models is shown in Figure 21 (left column for JI and right column for DS). Figure 21 shows that $80 \%$ of the CT scans had a JI of $0.94,0.96,0.97$, and 0.93 , respectively, for the four models. The DS for $80 \%$ of the CT scans had DS of 0.97, 0.97, 0.98, and 0.96, respectively for the four models SegNet, VGG-SegNet, ResNet-SegNet, and NIH conventional models.

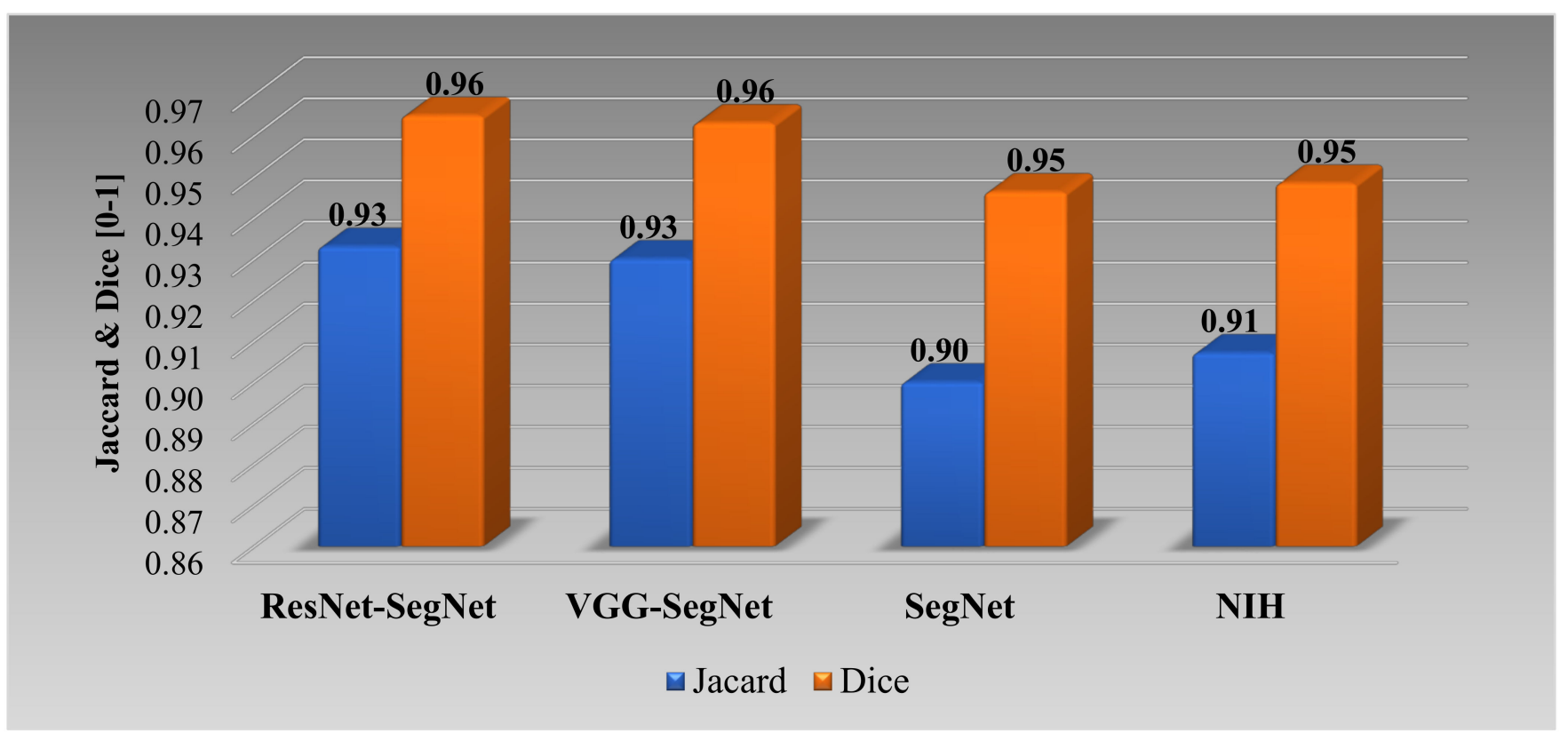

Figure 20. Bar Chart for JI and DS for four (two-hybrid, one deep, and NIH) models. 

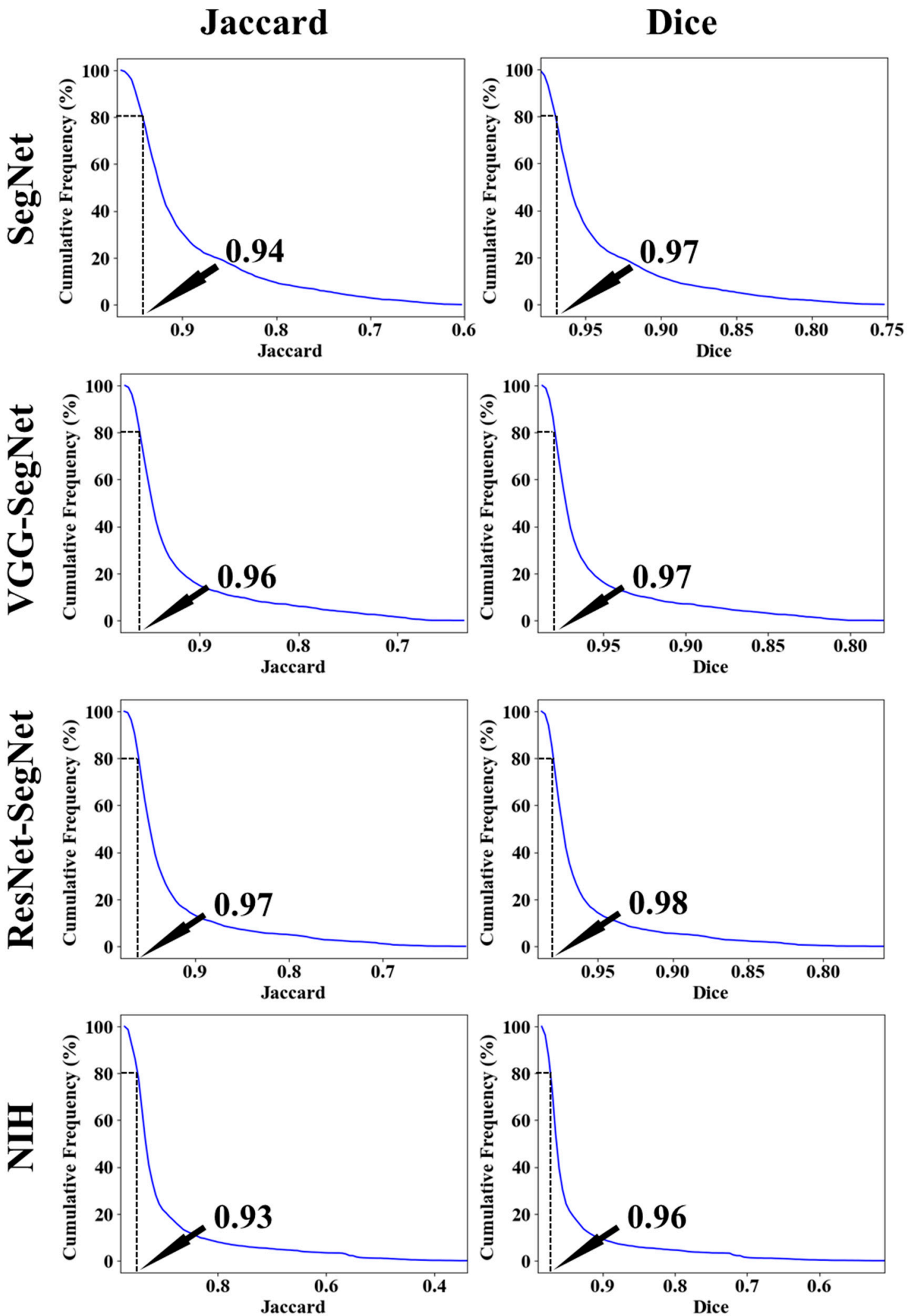

Figure 21. Cumulative frequency for JI and DS for the four models.

Bland-Altman Plot for Lung Area

A Bland-Altman plot is used to show the concurrency between two methods using the same variable. We follow the protocol for Bland-Altman computation based on our previous paradigms $[40,51]$. Figure 22 below shows the Bland-Altman plot by comput- 
ing (i) mean and (ii) difference between AI model area and GT area corresponding to 3000 COVID-19 test CT scans. The strongest concentration can be seen for the HDL models over the SDL model and NIH models. This is consistent with previous plots.

\section{Left Area}
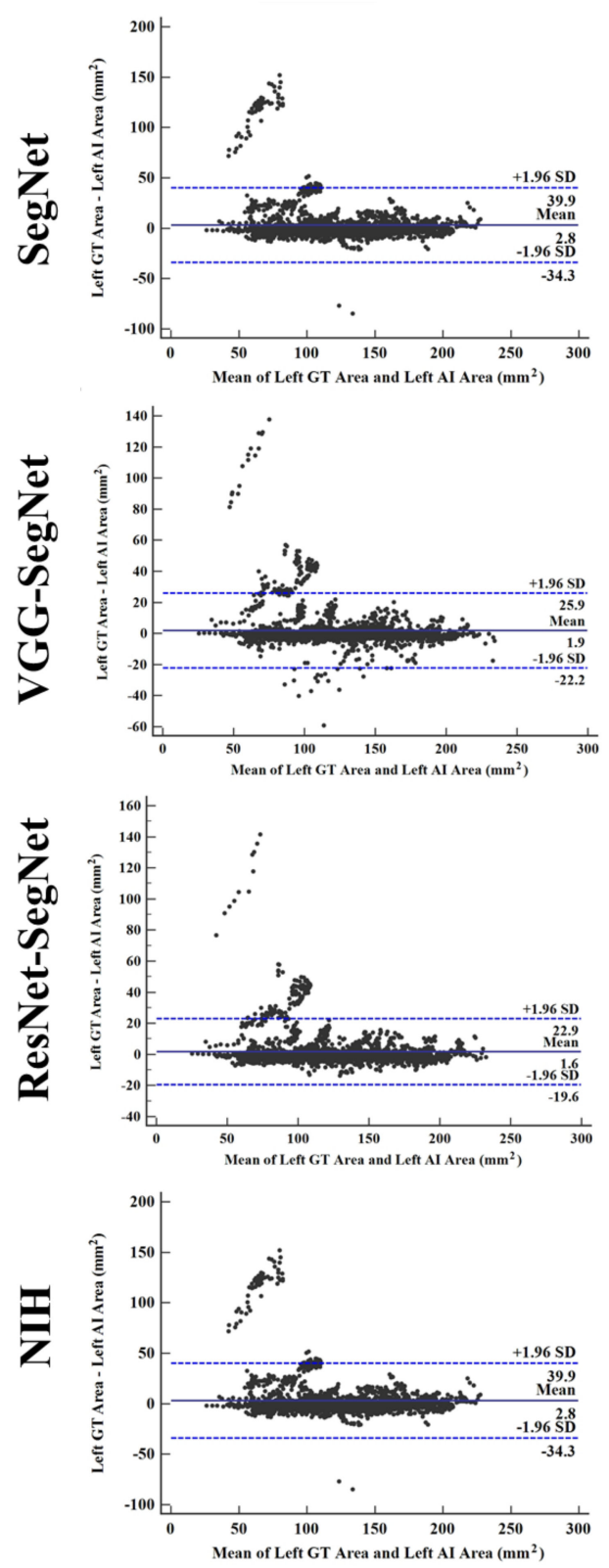

Right Area
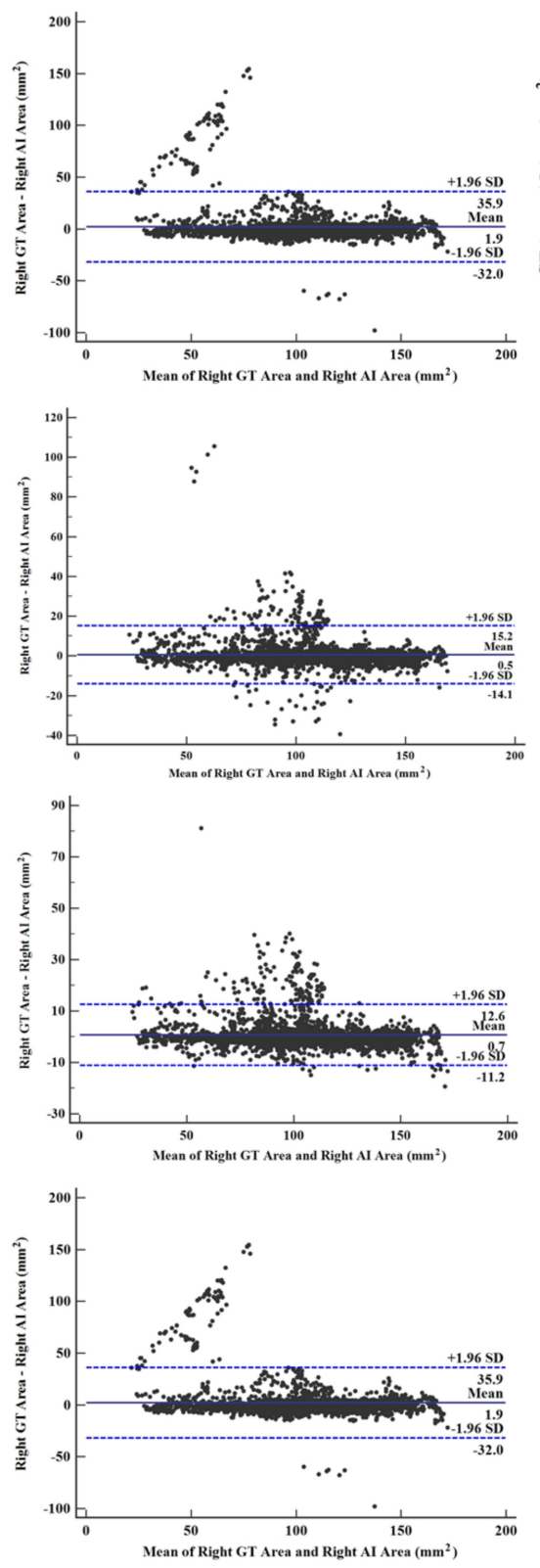

Mean Area
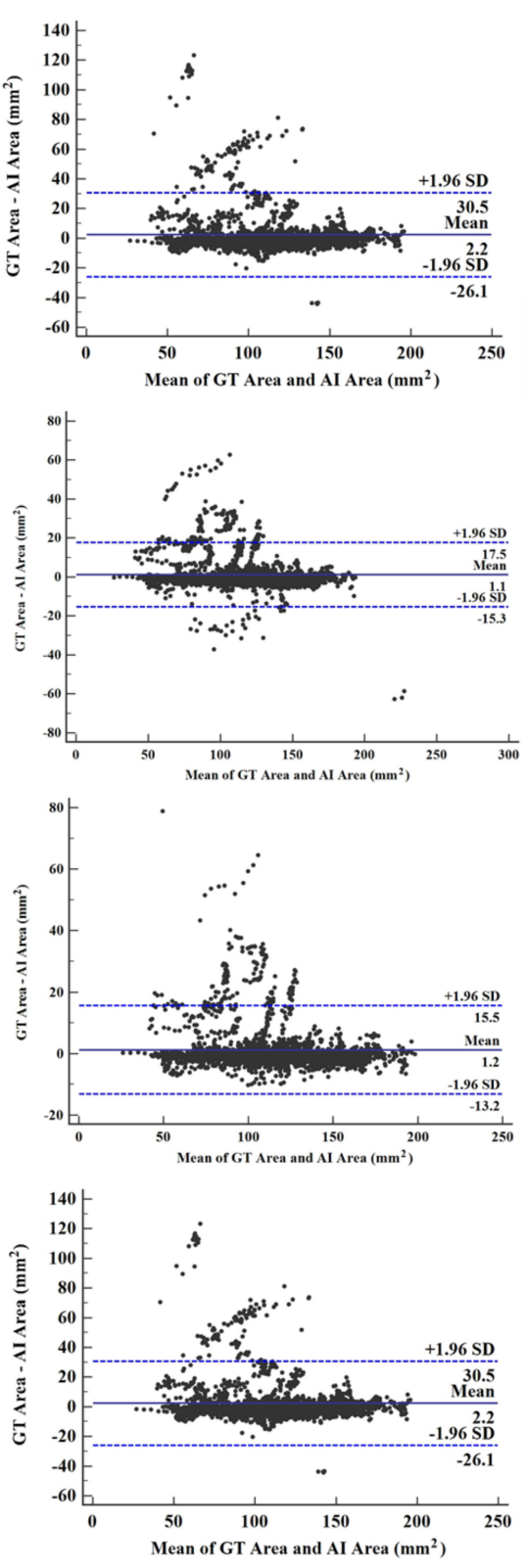

Figure 22. BA plot for LAE for the four models (rows) corresponding to left, right, and mean (columns).

\section{ROC Plots for Lung Area}

Figure 23 shows ROC plots for four models (rows) corresponding to left, right, and mean (columns). The corresponding AUC values, along with their $p$-values, are shown as well. All the AUCs were in the range of 0.96 to 0.99 ( $p$-value $<0.0001$ ). The highest performance was for ResNet-SegNet for the left, right, and mean lungs and given as 0.994, 0.974, and 0.987, respectively. The performance of the models for best to worst had the following order: ResNet-SegNet $>$ VGG-SegNet $>$ SegNet $>$ NIH. 

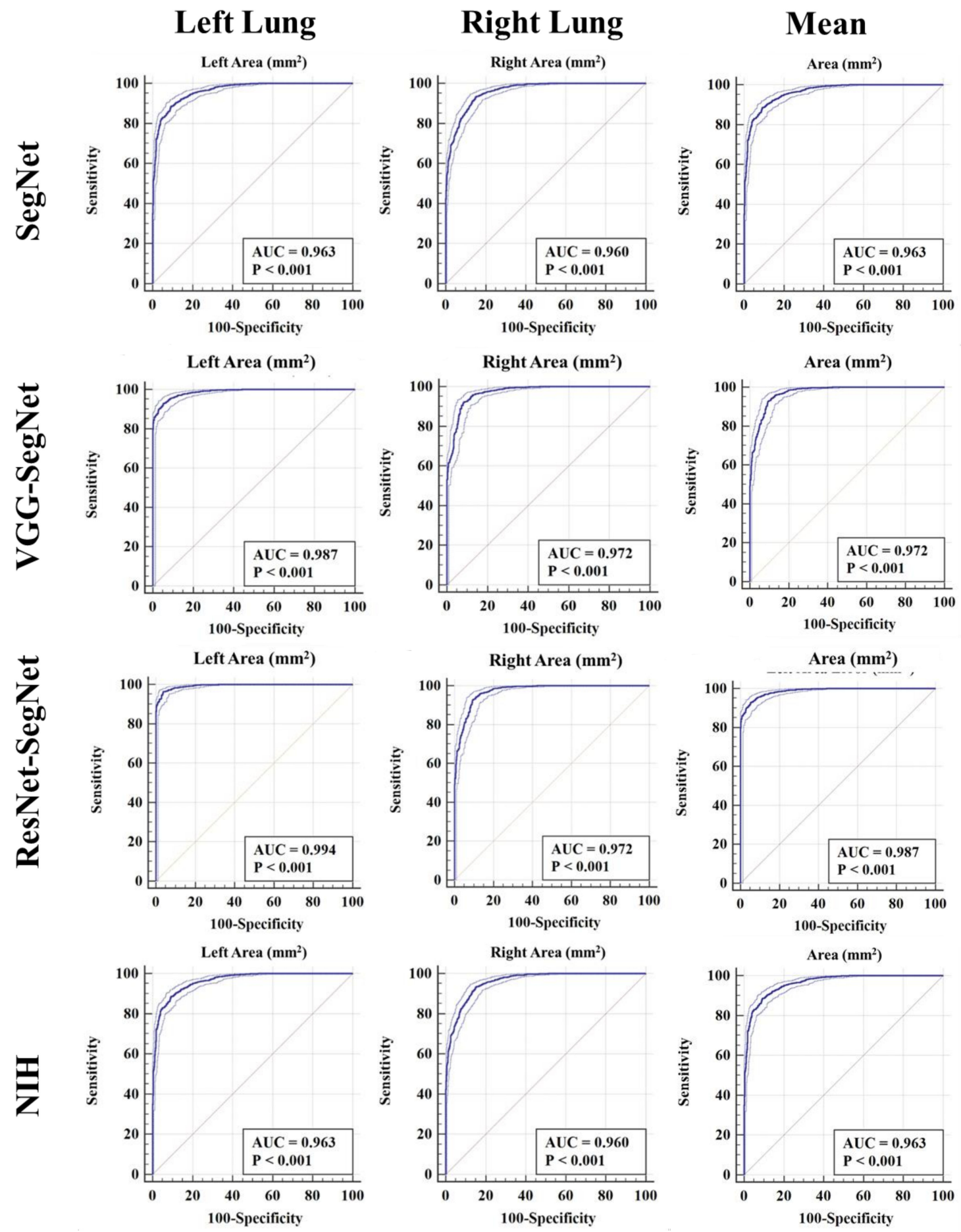

Figure 23. ROC plot for four models corresponding to left, right, and mean.

\subsubsection{Performance Evaluation Using Lung Long Axis Error}

Cumulative Frequency Plot for Lung Long Axis Error

Figure 24 shows the cumulative distribution of left and right LLAE over 3000 images from the test dataset. The cumulative frequency threshold of $80 \%$ was chosen to show the comparison amongst the three models. The corresponding bar charts for the LLAE using four models are shown in Figure 25. Decreasing error is shown from right to left, showing the performance of ResNet-SegNet, VGG-SegNet, SegNet, and NIH, which is consistent with area error in Section 4.2.2. 

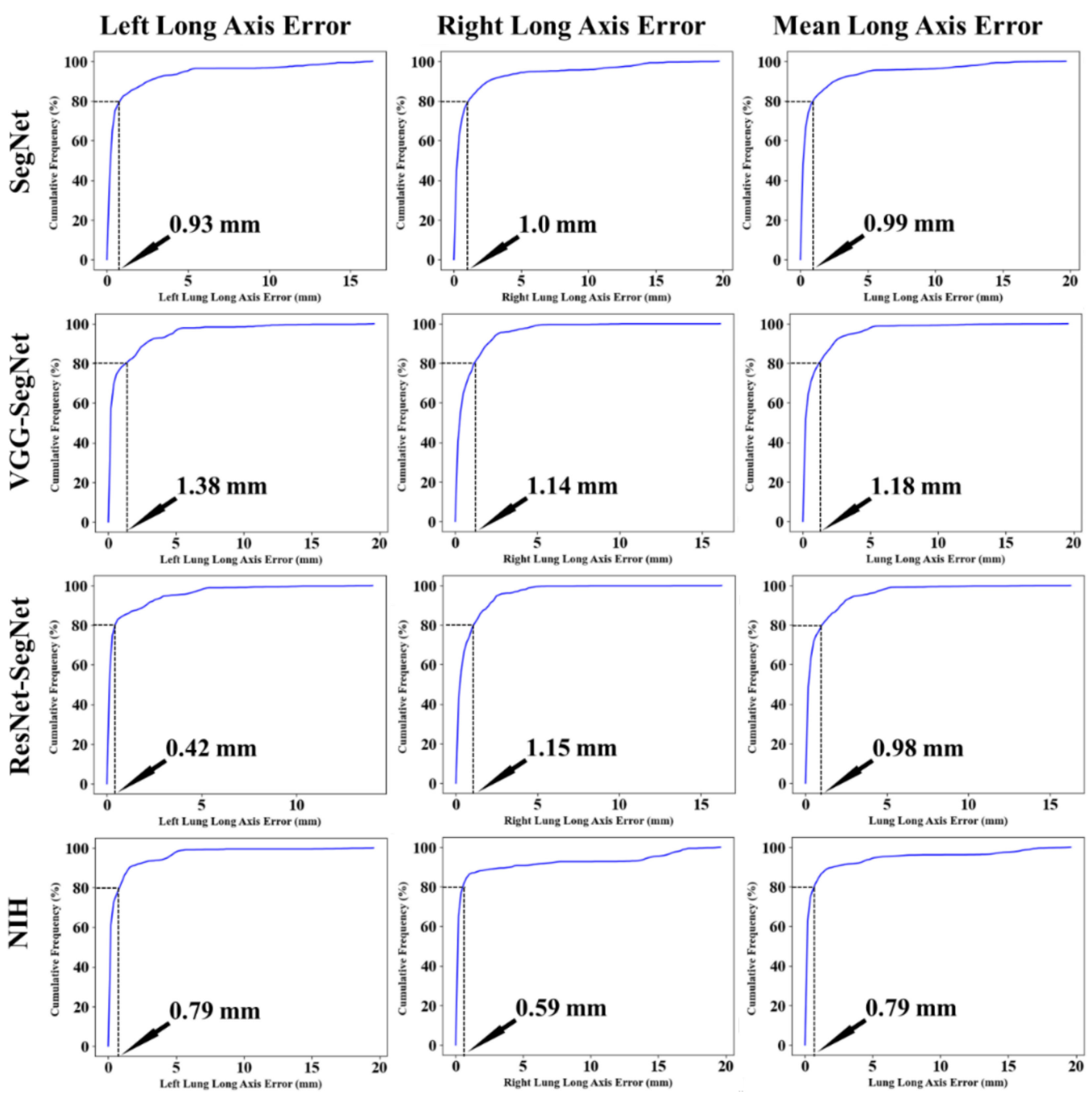

Figure 24. Cumulative plot for LLAE along with an $80 \%$ threshold for cumulative frequency for LLAE.

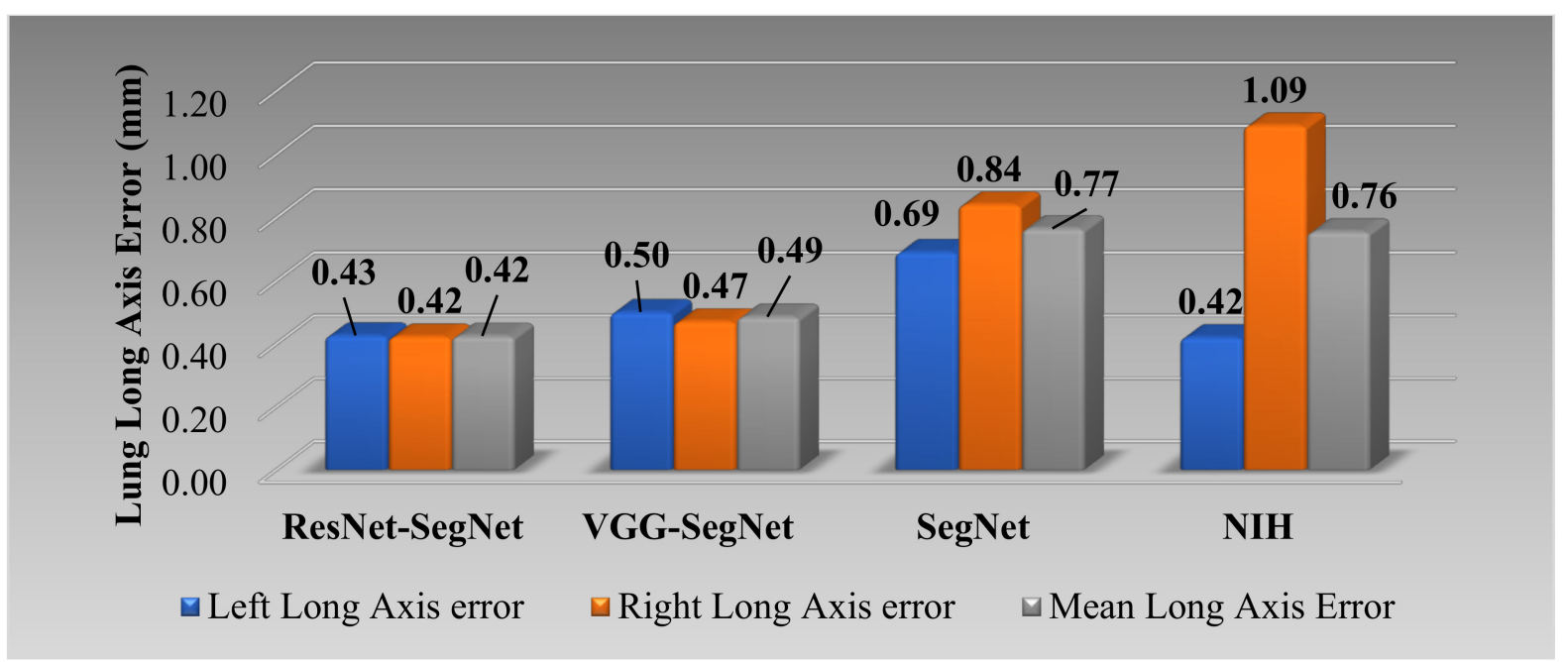

Figure 25. Bar chart for LLAE using four models.

Correlation Plot for Lung Long Axis Error

Figure 26 shows the correlation plots between lung long axes of the model vs. GT for the left, right, and mean. The CC varied from 0.90 to $0.98(p<0.0001)$. The order of the 
performance was from ResNet-SegNet, VGG-SegNet, SegNet, and NIH. The bar chart plot is shown in Figure 27, and the performance follows the pattern nearly the same order as ResNet-SegNet $>$ VGG-SegNet $>$ SegNet $>$ NIH.

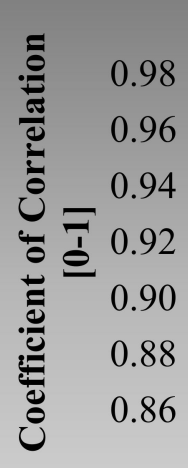

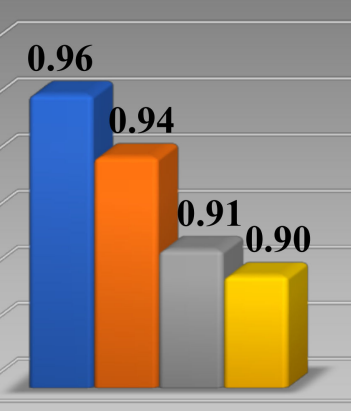

Left Long Axis
0.980 .97

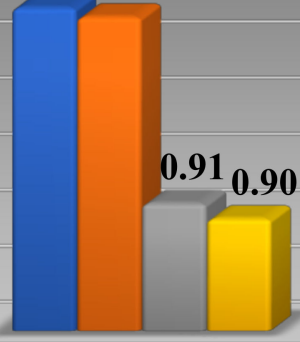

Right Long Axis

\subsection{0 .97}

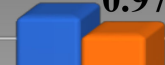

$\square$ ResNet-SegNet $\square$ VGG-SegNet $\square$ SegNet $\square$ NIH

Figure 26. Plot for correlation coefficient between the AI-based LLA vs. GT.

Left Long Axis Error
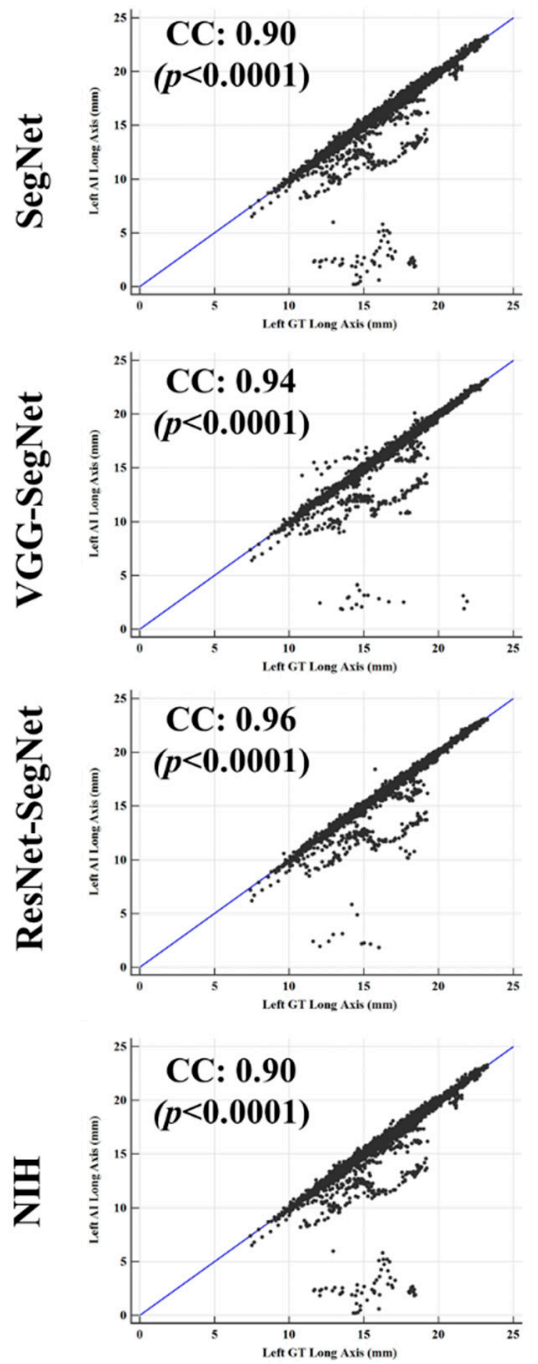
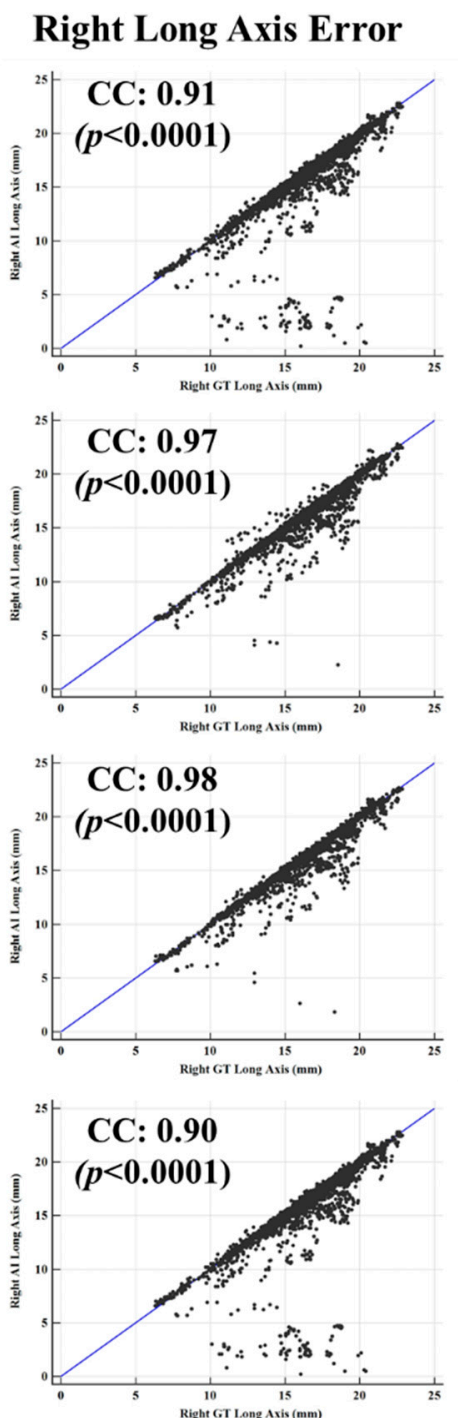
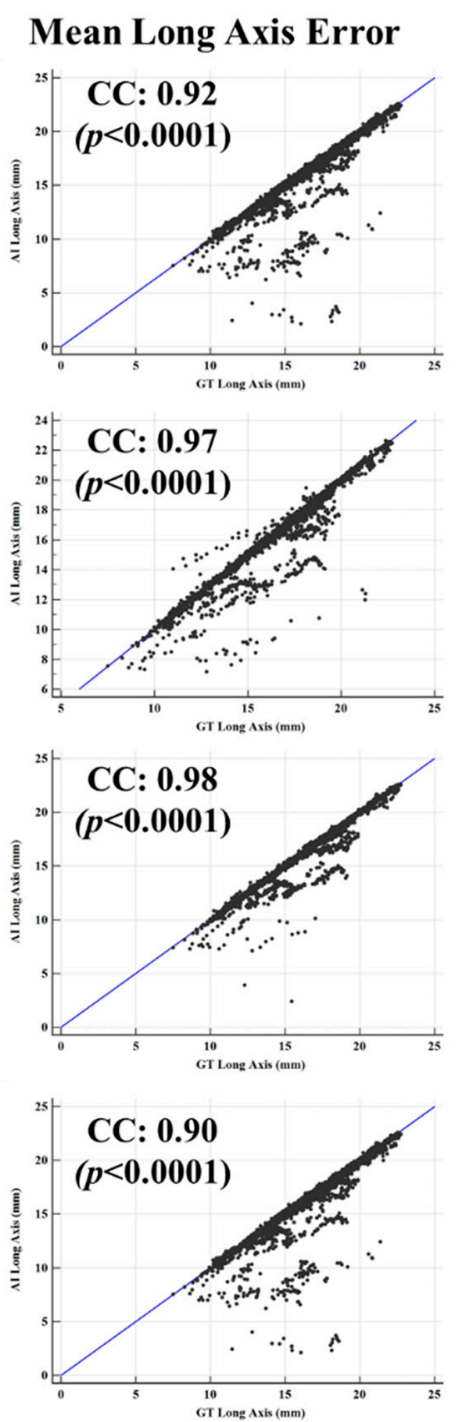

Figure 27. Correlation plot for LLA error for the four models corresponding to left, right, and mean. 
Bland-Altman Plots for Lung Long Axis Error

Figure 28 shows the Bland-Altman (BA) plot for the lung long axis for four models corresponding to left, right, and mean. The order of the performance was ResNet-SegNet, VGG-SegNet, SegNet, and NIH. This can be seen from the spread of the deviations.

\section{Left Long Axis}
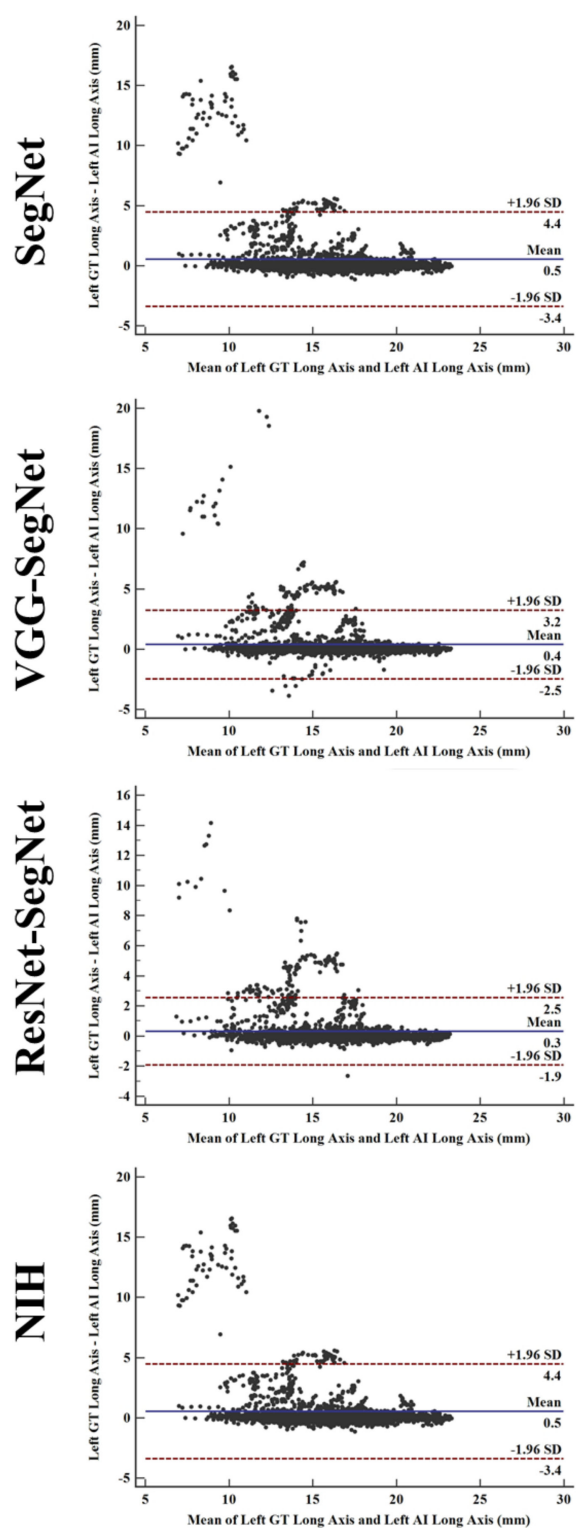

Right Long Axis
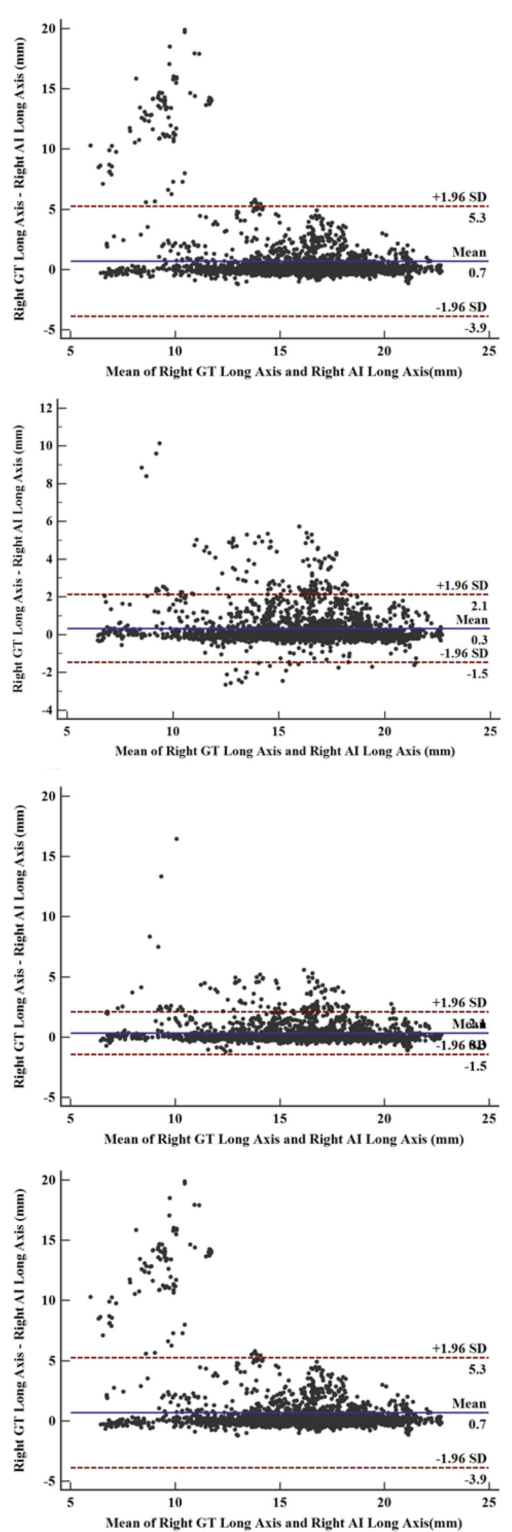

Mean Long Axis
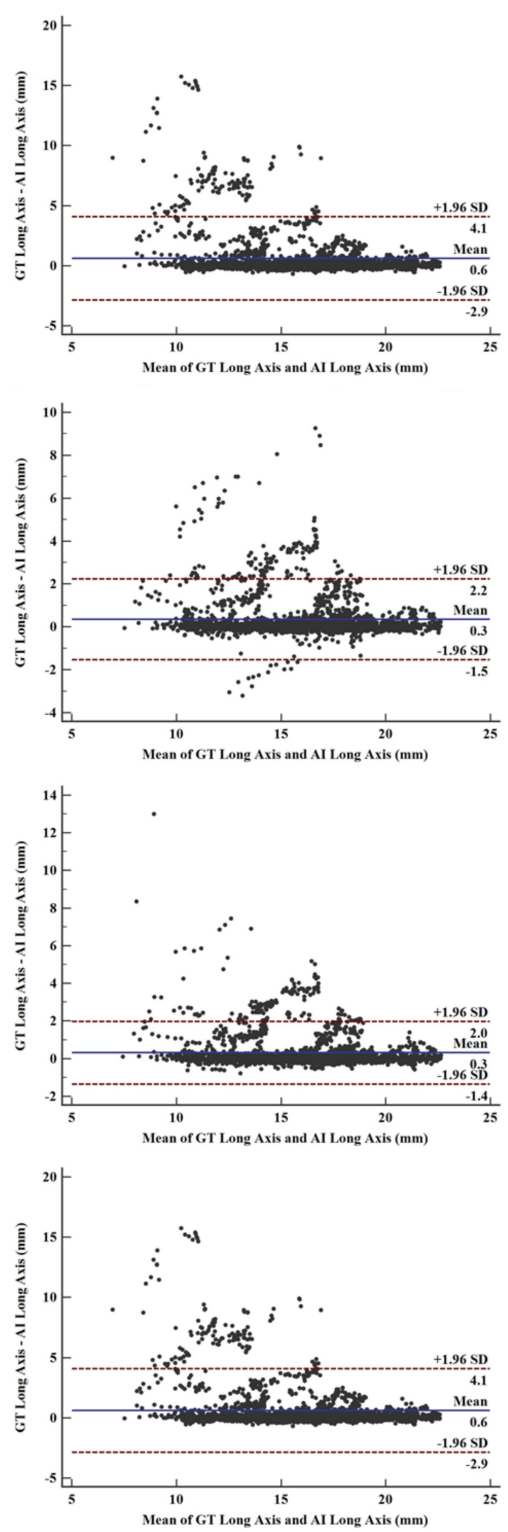

Figure 28. BA plot for LLA for four models corresponding to left, right, and mean.

The Figure of Merit Using Lung Area

The figure of merit $(F o M)$ is defined in terms of the central tendency of the error. Let $\mathcal{A}_{a i}(m, n)$ and $\mathcal{A}_{g t}(n)$ represent the lung area using AI model ' $m$ ' and GT, respectively, for the image ' $n$ '. Considering $N$ as the total number of CT scans, the corresponding mean AI for model $m$ and GT can be represented as $\overline{\mathcal{A}}_{a i}(m)$ and $\overline{\mathcal{A}}_{g t}$, as defined in Equation (3), and the FoM can be expressed in Equation (5). 
Table 1 shows the values for FoM for the models against the GT. The order of performance for the models is SegNet, VGG-SegNet, SegNet, and NIH:

$$
\operatorname{FoM}(m)=100-\left[\left(\frac{\left|\overline{\mathcal{A}}_{a i}(m)-\overline{\mathcal{A}}_{g t}\right|}{\overline{\mathcal{A}}_{g t}}\right) * 100\right],
$$

where $\overline{\mathcal{A}}_{a i}(m)=\frac{\sum_{n=1}^{N} \mathcal{A}_{a i}(m, n)}{N} \& \overline{\mathcal{A}}_{g t}=\frac{\sum_{n=1}^{N} \mathcal{A}_{g t}(n)}{N}$.

Table 1. FoM for the segmentation modes for lung area and lung long axis.

\begin{tabular}{ccccccc}
\hline & \multicolumn{3}{c}{ Lung Area } & \multicolumn{3}{c}{ Lung Long Axis } \\
\cline { 2 - 7 } & Left & Right & Mean & Left & Right & Mean \\
\hline SegNet & 94.28 & 97.6 & 95.94 & 97.03 & 98.53 & 97.78 \\
\hline VGG-SegNet & 99.09 & 99.56 & 99.32 & 99.7 & 99.61 & 99.65 \\
\hline ResNet-SegNet & 99.03 & 99.98 & 99.55 & 99.72 & 99.62 & 99.67 \\
\hline NIH & 94.98 & 98.69 & 96.83 & 97.76 & 96.09 & 96.92 \\
\hline
\end{tabular}

Percentage Improvement of AI Models against the NIH Model Using the Lung Area and Lung Long Axis.

This performance measure shows the improvement of the AI models against the NIH, the so-called benchmarking solution (Table 2).

Table 2. Improvement of three AI models against the NIH model for lung area and lung long axis.

\begin{tabular}{ccccccc}
\hline & \multicolumn{3}{c}{ Lung Area Error } & \multicolumn{3}{c}{ Lung Long Axis Error } \\
\cline { 2 - 7 } & Left Lung & Right Lung & Mean & Left Lung & Right Lung & Mean \\
\hline ResNet-SegNet & $57 \%$ & $60 \%$ & $58 \%$ & $42 \%$ & $44 \%$ & $43 \%$ \\
\hline VGG-SegNet & $51 \%$ & $52 \%$ & $52 \%$ & $31 \%$ & $37 \%$ & $34 \%$ \\
\hline SegNet & $22 \%$ & $19 \%$ & $21 \%$ & $5 \%$ & $13 \%$ & $4 \%$ \\
\hline
\end{tabular}

\section{Discussion}

This study presented a COVLIAS 1.0 system that consisted of two hybrid deep learning and one solo deep learning artificial intelligence model for lung segmentation in COVID-19 CT lung scans. The system was then compared against the conventional NIH-released model. Seventy-two COVID-19 patients were considered for the study, which consisted of 5000 CT scans. Cross-validation protocol was implemented using $40 \%$ training and $60 \%$ testing data sets. The performance of the system was computed using three main kinds of metrics. This consisted of two sets of visualization components: (i) visualization of the model boundary against the ground truth boundary with different boundary colors; and (ii) visualization of the superimposition of the segmented model lung region against the GT lung region with different colors; the second set of performance evaluation was based on the LAE and consisted of (i) cumulative plot of the LAE; (ii) Bland-Altman plot for the LAE; (iii) JI and DS for the lung region; and (iv) ROC curves for AI-based model performance. The third set of performance evaluation was based on the LLAE and consisted of the following components: (I) cumulative plot of the LLAE; (ii) correlation coefficient of the lung long axis; (iii) correlation coefficient of the lung long axis error; and (iv) Bland-Altman plot for the LLAE. In all the metrics, HDL performed better than SDL and both were better than the conventional NIH model, retaining the order of ResNet-SegNet > VGGSegNet $>$ SegNet $>$ NIH. Note that the COVILAS 1.0 system (AtheroPoint ${ }^{\mathrm{TM}}$, Roseville, CA, USA) was designed using Python language unlike other tools typically designed in MATLAB. The COVLIAS 1.0 used solo deep learning and hybrid deep learning solutions for lung segmentation for CT lung COVID-19 scans. The Python system was developed in a Windows 10 environment, while the graphical user interface was designed using JavaScript. It adapts multitasking and multithreaded architecture for COVLIAS 1.0. 


\subsection{Benchmarking}

Several studies have been published with deep learning systems based on chest CT imaging to distinguish COVID-19 cases from non-COVID-19 cases (which may include normal and abnormal cases) and segment the COVID-19 region from the lungs [52-55]. However, most of them do not include lung segmentation, boundary estimation, or lung region mask overlays compared to GT. Table 3 shows the benchmarking table showing five studies: Priya et al. (2021) [56], Saood et al. (2021) [57], Paluru et al. (2020) [58], and Cai et al. (2020) [59], which focused on lung segmentation. The table also compares against the proposed study by Suri et al. (2021). Cai et al. [59] have used a deep learning-based UNet model to segment the lung and show the boundary of the segmented lung with a DSC of 0.98 and Jaccard of 0.96. Xiao et al. [60] have demonstrated CNN architecture for segmentation of lung and showed a DSC of 0.98 . Our study used a hybrid deep learning AI solution for computing lung region with a study database of $5000 \mathrm{CT}$ scans demonstrating DS of $0.97,0.97,0.98$, and 0.96 . The JI for the four models were $0.94,0.96,0.97$, and 0.93 , respectively. The AUC for the four models were $0.96,0.96,0.97$, and 0.98 , respectively, the highest of the previous methods. The accuracies of the proposed AI models were $98 \%, 98 \%$, and $99 \%$, respectively. Overall, the proposed study offers a first-time hybrid solution in conjunction with solo deep learning and benchmarking against the NIH-based approach, proving our hypothesis that hybrid deep learning artificial intelligence models are superior to solo deep learning models for COVID-19 lung segmentation.

\subsection{A Special Note on the HDL Model}

Our results show that HDL is superior to SDL, and both were superior to the conventional NIH model. HDL takes advantage of combining the two SDL models. Several studies were published in the image classification area that showed HDL was superior to SDL. EL-Kenawy et al. [61] used Stochastic Fractal Search (SFS) combined with the Whale Optimization Algorithm (WOA) as part of the HDL, and the authors showed superior results compared to SDL models such as AlexNet, VGGNet16, VGGNet19, GoogleNet, and ResNet50. Vijayalakshmi et al. [62] implemented an HDL model where the authors combined SVM with VGG16 and showed superior results compared to SDL such as VGG16 or VGG19.

\subsection{A Note on Comparison between the Proposed AI and NIH Conventional Models}

The fundamental difference between the proposed AI models and the NIH model is the role of feature extraction during the lung segmentation process. The feature extraction step is the most vital component of the entire system. This component is accomplished automatically in the AI model, unlike the semi-automatically implemented in the NIH model. This automaticity provides considering time-saving in AI models, unlike in the conventional NIH model. Second, the NIH model has several sub-systems which are user-controlled, such as seed point selection, and image format conversion from High dynamic range (HDR) to Neuroimaging Informatics Technology Initiative (NII/NIfTI) to Portable Network Graphics (PNG) format. It is very tedious and time-consuming when you have to execute this on $5000 \mathrm{CT}$ slices. This is where the AI system comes in handy, is time saving, and is efficient. Our analysis showed that the AI model typically takes one second on any test CT slice, unlike in the NIH model, which took $10 \mathrm{~min}$ for a single slice. Note that the NIH model required that the user inputs the entire CT volume of the patient for segmenting the lungs in the $\mathrm{CT}$ scans. The processing time for the volume of interest consisting of typically 60 selected slices took $\sim 20 \mathrm{~min}$ based on the hardware configuration. This included uploading of volumes, semi-automated segmentation, format conversion, and saving of all the segmented results. 
Table 3. Benchmarking table.

\begin{tabular}{|c|c|c|c|c|c|c|c|c|c|c|c|c|c|c|c|c|c|}
\hline $\begin{array}{l}\text { Author } \\
\text { (Year) }\end{array}$ & $\begin{array}{c}\text { \# of } \\
\text { Patients }\end{array}$ & Demo. & $\begin{array}{c}\text { \# of } \\
\text { Images }\end{array}$ & IS $^{2}$ & $\begin{array}{c}\text { \# of } \\
\text { Tracers }\end{array}$ & $\begin{array}{c}\text { AI } \\
\text { Model }\end{array}$ & $\begin{array}{l}\text { SDL vs. } \\
\text { HDL }\end{array}$ & Dim. & $\begin{array}{c}\text { Training } \\
\text { Speed }\end{array}$ & Heatmap & VS & $\mathrm{AE}$ & BE & DS & JI & AUC & $\begin{array}{c}\text { ACC } \\
(\%)\end{array}$ \\
\hline $\begin{array}{l}\text { Priya et al. } \\
\text { (2021) [56] }\end{array}$ & - & No & 78 & - & - & $\begin{array}{c}\text { Squeeze } \\
\text { Net }\end{array}$ & HDL & $2 \mathrm{D}$ & - & Yes & No & - & Yes & - & - & - & - \\
\hline $\begin{array}{l}\text { Saood et al. } \\
\text { (2021) [57] }\end{array}$ & - & No & 100 & $256^{2}$ & - & $\begin{array}{c}\text { UNet, } \\
\text { SegNet }\end{array}$ & SDL & $2 \mathrm{D}$ & - & - & No & - & - & $\begin{array}{l}0.73, \\
0.74\end{array}$ & - & $\begin{array}{l}0.94 \\
0.95\end{array}$ & $\begin{array}{l}91, \\
95\end{array}$ \\
\hline $\begin{array}{c}\text { Paluru et al. } \\
(2020) \text { [58] }\end{array}$ & 69 & No & 4339 & $512^{2}$ & - & Anam-net & SDL & $2 \mathrm{D}$ & $27 \mathrm{~min}$ & - & Yes & - & - & 0.75 & - & 0.99 & 99.1 \\
\hline $\begin{array}{l}\text { Cai et al. } \\
\text { (2020) [59] }\end{array}$ & 99 & Yes & 6301 & & 2 & UNet & SDL & $2 \mathrm{D}$ & - & yes & No & - & - & 0.98 & 0.96 & - & - \\
\hline $\begin{array}{c}\text { Suri et al. } \\
\text { (2021) }\end{array}$ & 72 & Yes & 5000 & $768^{2}$ & 1 & $\begin{array}{c}\text { NIH, } \\
\text { SegNet, } \\
\text { VGG- } \\
\text { SegNet, } \\
\text { ResNet- } \\
\text { SegNet }\end{array}$ & $\begin{array}{c}\text { SDL } \\
\text { and HDL }\end{array}$ & $2 \mathrm{D}$ & $18 \mathrm{~min}$ & No & No & Yes & Yes & $\begin{array}{l}0.96 \\
0.97 \\
0.97 \\
0.98\end{array}$ & $\begin{array}{l}0.93 \\
0.94 \\
0.96 \\
0.97\end{array}$ & $\begin{array}{l}0.96 \\
0.96 \\
0.97 \\
0.98\end{array}$ & $\begin{array}{c}\text { NA, } \\
98, \\
98, \\
99\end{array}$ \\
\hline
\end{tabular}

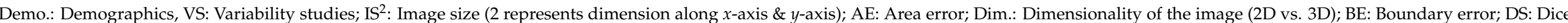
similarity; JI: Jaccard index; AUC: Area under the curve; SDL: Solo deep learning; HDL: Hybrid deep learning; ACC: Accuracy. 


\subsection{Strength, Weakness, and Extension}

The study presented two HDL models, one SDL model, and one conventional NIH model for lung segmentation in COVID-19 CT lungs for a cohort of 5000 images. The results of the study showed consistency where HDL models were superior to SDL models. The online system took less than $1 \mathrm{~s}$.

Even though the COVLIAS 1.0 was the first of its kind where HDL was designed and developed, more validation is needed for conduction involving multicenter studies $[39,63]$. Furthermore, cyclic cross-validation needs to be conducted for further comparisons [64,65]. The system can be extended to estimate COVID-19 severity in the lung region while keeping the services cost-effective [66]. Variability analysis can also be conducted where multiple tracers can be involved [40]. The COVLIAS 1.0 is a pilot study with encouraging results. We intend to pursue more $\mathrm{CV}$ protocols with large data size and more models in the big data framework [67] in the future.

Comorbidity is an essential factor that has been linked to COVID-19 right from the beginning. Recently, a study was conducted that showed the relation of the effect of the comorbidity on AI model design [6]. In our study, the patients had no prior issues before getting admitted to Novara hospital, Italy during a pandemic. In some cases, however, it is possible that the patients had concomitant or incidental findings such as scars or small nodules. The scope of this study did not incorporate such incidental findings enough to incorporate them into the AI model. This could potentially be a future possibility of more sophisticated data collection and modeling of AI. Furthermore, the COVLIAS 1.0 system can be extended to other kinds of pneumonia, interstitial lung diseases, and cancer $[25,68]$. Lastly, the AI model can be compared with conventional models as previously attempted applications [69].

\section{Conclusions}

The study presented four kinds of lung segmentation methods for COVID-19 CT lung scans under the COVLIAS 1.0 (AtheroPoint ${ }^{\mathrm{TM}}$, Roseville, CA, USA). It consisted of three AI-based models: two-hybrid deep learning (HDL) models and one SDL. These three AI models were benchmarked against the NIH-based conventional model. The COVILAS 1.0 used a database of 5000 COVID-19 CT images for designing the cross-validation protocol that consisted of 4:6 ratios for training to testing with validation data.

Comprehensive performance matrices were designed such as (i) visualization of the model boundary against the ground truth boundary with different boundary colors; (ii) visualization of the superimposition of the model lung region against the GT lung region with different colors; (iii) performance evaluation based on the LAE and consisted of (a) cumulative plot of the lung area error; (b) Bland-Altman plot for the lung area error; (c) Jaccard index and dice similarity for the lung region; and (d) ROC curves for AI-based model performance. (iv) performance evaluation based on the lung long axis error and consists of the following components (a) cumulative plot of the lung long axis error; (b) correlation coefficient of the lung long axis error; and (c) Bland-Altman plot for the lung long axis error.

We further conclude that the AI-based models were better than the conventional NIH model, and HDL was superior to SDL. The order of performance for all metrics were ResNet-SegNet > VGG-SegNet $>$ SegNet $>$ NIH. The COVLIAS 1.0 system is currently under evaluation by radiology departments at friendly sites. 
Author Contributions: Conceptualization: J.S.S., M.K., N.N.K. and M.M.; Data Curation: M.C., L.S., G.F., M.T., K.V., P.R.K., N.F., Z.R. and A.G.; Formal Analysis: J.S.S. and M.K.; Investigation: J.S.S., M.K., I.M.S., P.S.C., A.M.J., S.M., J.R.L., G.P., D.W.S., P.P.S., G.T., A.P., D.P.M., V.A., J.S.T., M.A.-M., S.K.D., A.N., A.S. and A.A.; Methodology: J.S.S., S.A. and A.B.; Project Administration: J.S.S. and M.K.; Deep Learning Computing Resources: S.K.G.; Software Design and Usage: S.A. and V.K.; Software Verification: J.S.S., L.S. and M.K.; Supervision: J.S.S., M.K. and S.N.; Scientific Validation: J.S.S. and S.A.; Clinical Validation and Discussions: J.S.S., M.K. and L.S.; Visualization: S.A.; WritingOriginal Draft: J.S.S. and S.A.; Writing-Review and Editing: J.S.S., M.K., L.S., R.P., S.K.G., I.M.S., M.T., P.S.C., A.M.J., N.N.K., K.V., S.M., J.R.L., G.P., M.M., D.W.S., A.B., P.P.S., G.T., A.P., D.P.M., V.A., G.D.K., M.A.-M., S.K.D., A.N., A.S., V.R., M.F., N.F., A.G. and S.N. All authors have read and agreed to the published version of the manuscript.

Funding: This research received no external funding.

Institutional Review Board Statement: Not applicable.

Informed Consent Statement: Not applicable.

Data Availability Statement: Not applicable.

Conflicts of Interest: The authors declare no conflict of interest.

\section{Acronym Table}

$\begin{array}{lll}\text { SN } & \text { Symbol } & \text { Description of the Symbols } \\ 1 & \text { ACC } & \text { Accuracy } \\ 2 & \text { AE } & \text { Area Error } \\ 3 & \text { AI } & \text { Artificial Intelligence } \\ 4 & \text { ARDS } & \text { Acute Respiratory Distress Syndrome } \\ 5 & \text { AUC } & \text { Area Under the Curve } \\ 6 & \text { BA } & \text { Bland-Altman } \\ 7 & \text { BE } & \text { Boundary Error } \\ 8 & \text { CC } & \text { Correlation coefficient } \\ 9 & \text { CE } & \text { Cross Entropy } \\ 10 & \text { COVID } & \text { Coronavirus disease } \\ 11 & \text { COVLIAS } & \text { COVID Lung Image Analysis System } \\ 12 & \text { CT } & \text { Computed Tomography } \\ 13 & \text { DL } & \text { Deep Learning } \\ 14 & \text { DS } & \text { Dice Similarity } \\ 15 & \text { FoM } & \text { Figure of merit } \\ 16 & \text { GT } & \text { Ground Truth } \\ 17 & \text { HDL } & \text { Hybrid Deep Learning } \\ 18 & \text { IS } & \text { Image Size } \\ 19 & \text { JI } & \text { Jaccard Index } \\ 20 & \text { LAE } & \text { Lung Area Error } \\ 21 & \text { LLAE } & \text { Lung Long Axis Error } \\ 22 & \text { NIH } & \text { National Institute of Health } \\ 23 & \text { PC } & \text { Pixel Counting } \\ 24 & \text { RF } & \text { Resolution Factor } \\ 25 & \text { ROC } & \text { Receiver operating characteristic } \\ 26 & \text { SDL } & \text { Solo Deep Learning } \\ 27 & \text { VGG } & \text { Visual Geometric Group } \\ 28 & \text { VS } & \text { Variability studies } \\ 29 & \text { WHO } & \text { World Health Organization } \\ & & \end{array}$




\section{Symbol Table}

\begin{tabular}{|c|c|c|}
\hline$\overline{\mathrm{SN}}$ & Symbol & Description of the Symbols \\
\hline 1 & $L_{C E}$ & Cross Entropy-loss \\
\hline 2 & $L_{D S C}$ & Dice Similarity Coefficient-loss \\
\hline 3 & $m$ & Model number used for segmentation in the total number of models $\mathrm{M}$ \\
\hline 4 & $n$ & Image scan number a total number of image N. \\
\hline 5 & $\overline{\mathcal{A}}_{a i}(m)$ & Mean estimated lung area for all images using AI model ' $m$ ' \\
\hline 6 & $\mathcal{A}_{a i}(m, n)$ & Estimated Lung Area using AI model ' $m$ ' and image ' $n$ ' \\
\hline$\underline{7}$ & $\mathcal{A}_{g t}(n)$ & GT lung area for image ' $n$ ' \\
\hline 8 & $\overline{\mathcal{A}_{g t}}$ & Mean ground truth area for all images $\mathrm{N}$ in the database \\
\hline 9 & $\operatorname{FoM}(m)$ & Figure-of-Merit for segmentation model ' $m$ ' \\
\hline 10 & $\mathrm{JI}$ & Mean Jaccard Index for a specific segmentation model \\
\hline 11 & DSC & Dice Similarity Coefficient for a specific segmentation model \\
\hline 12 & $N_{p}$ & Sample size required computed using power analysis \\
\hline 13 & MoE & Margin-of-Error \\
\hline 14 & $\mathrm{TP}, \mathrm{TN}$ & True Positive and True Negative \\
\hline 15 & $\mathrm{FP}, \mathrm{FN}$ & False Positive and False Negative \\
\hline 16 & $\mathrm{y}_{\mathrm{i}}$ & GT label \\
\hline 17 & $\mathrm{a}_{\mathrm{i}}$ & SoftMax classifier probability \\
\hline 18 & $\mathrm{Y}_{\mathrm{p}}$ & Ground truth image \\
\hline 19 & $\hat{Y}_{p}$ & Estimated image \\
\hline 20 & $\mathrm{P}$ & Total number of pixels in an image in $x, y$-direction \\
\hline 21 & $\mathrm{z}$ & Z-score from standard z-table \\
\hline 22 & K5-r46 & Cross-validation protocol with $40 \%$ training and $60 \%$ testing \\
\hline \multicolumn{3}{|c|}{ Deep Learning Segmentation Architectures } \\
\hline 23 & SegNet & SDL model for lung segmentation with reduced learning parameters \\
\hline 24 & VGG-SegNet & HDL model designed by fusion of VGG-19 and SegNet architecture \\
\hline 25 & ResNet-SegNet & HDL model designed by fusion of ResNet-50 and SegNet architecture \\
\hline \multicolumn{3}{|c|}{ Conventional Model } \\
\hline 26 & $\mathrm{NIH}$ & National Institute of Health segmentation model \\
\hline
\end{tabular}

\section{References}

1. WHO Coronavirus (COVID-19) Dashboard. Available online: https:/ / covid19.who.int/ (accessed on 28 July 2021).

2. Saba, L.; Gerosa, C.; Fanni, D.; Marongiu, F.; La Nasa, G.; Caocci, G.; Barcellona, D.; Balestrieri, A.; Coghe, F.; Orru, G.; et al. Molecular pathways triggered by COVID-19 in different organs: ACE2 receptor-expressing cells under attack? A review. Eur. Rev. Med. Pharmacol. Sci. 2020, 24, 12609-12622. [CrossRef]

3. Cau, R.; Bassareo, P.P.; Mannelli, L.; Suri, J.S.; Saba, L. Imaging in COVID-19-related myocardial injury. Int. J. Cardiovasc. Imaging 2021, 37, 1349-1360. [CrossRef] [PubMed]

4. Viswanathan, V.; Puvvula, A.; Jamthikar, A.D.; Saba, L.; Johri, A.M.; Kotsis, V.; Khanna, N.N.; Dhanjil, S.K.; Majhail, M.; Misra, D.P. Bidirectional link between diabetes mellitus and coronavirus disease 2019 leading to cardiovascular disease: A narrative review. World J. Diabetes 2021, 12, 215. [CrossRef] [PubMed]

5. Cau, R.; Pacielli, A.; Fatemeh, H.; Vaudano, P.; Arru, C.; Crivelli, P.; Stranieri, G.; Suri, J.S.; Mannelli, L.; Conti, M.; et al. Complications in COVID-19 patients: Characteristics of pulmonary embolism. Clin. Imaging 2021, 77, 244-249. [CrossRef] [PubMed]

6. Suri, J.S.; Agarwal, S.; Gupta, S.K.; Puvvula, A.; Biswas, M.; Saba, L.; Bit, A.; Tandel, G.S.; Agarwal, M.; Patrick, A. A narrative review on characterization of acute respiratory distress syndrome in COVID-19-infected lungs using artificial intelligence. Comput. Biol. Med. 2021, 130, 104210. [CrossRef]

7. Cau, R.; Falaschi, Z.; Paschè, A.; Danna, P.; Arioli, R.; Arru, C.D.; Zagaria, D.; Tricca, S.; Suri, J.S.; Karla, M.K. Computed tomography findings of COVID-19 pneumonia in Intensive Care Unit-patients. J. Public Health Res. 2021, 2019. [CrossRef]

8. El-Baz, A.; Suri, J. Lung Imaging and CADx; CRC Press: Boca Raton, FL, USA, 2019.

9. $\quad$ El-Baz, A.; Suri, J.S. Lung Imaging and Computer Aided Diagnosis; CRC Press: Boca Raton, FL, USA, 2011.

10. Saba, L.; Suri, J.S. Multi-Detector CT Imaging: Principles, Head, Neck, and Vascular Systems; CRC Press: Boca Raton, FL, USA, 2013; Volume 1. 
11. Saba, L.; Agarwal, M.; Patrick, A.; Puvvula, A.; Gupta, S.K.; Carriero, A.; Laird, J.R.; Kitas, G.D.; Johri, A.M.; Balestrieri, A.; et al. Six artificial intelligence paradigms for tissue characterisation and classification of non-COVID-19 pneumonia against COVID-19 pneumonia in computed tomography lungs. Int. J. Comput. Assist. Radiol. Surg. 2021, 16, 423-434. [CrossRef]

12. Saba, L.; Biswas, M.; Suri, H.S.; Viskovic, K.; Laird, J.R.; Cuadrado-Godia, E.; Nicolaides, A.; Khanna, N.; Viswanathan, V.; Suri, J.S.J.C.d.; et al. Ultrasound-based carotid stenosis measurement and risk stratification in diabetic cohort: A deep learning paradigm. Cardiovasc. Diagn. Ther. 2019, 9, 439. [CrossRef]

13. Biswas, M.; Kuppili, V.; Saba, L.; Edla, D.R.; Suri, H.S.; Cuadrado-Godia, E.; Laird, J.R.; Marinhoe, R.T.; Sanches, J.M.; Nicolaides, A.; et al. State-of-the-art review on deep learning in medical imaging. Front. Biosci. 2019, 24, 392-426.

14. Saba, L.; Biswas, M.; Kuppili, V.; Cuadrado Godia, E.; Suri, H.S.; Edla, D.R.; Omerzu, T.; Laird, J.R.; Khanna, N.N.; Mavrogeni, S.; et al. The present and future of deep learning in radiology. Eur. J. Radiol. 2019, 114, 14-24. [CrossRef]

15. El-Baz, A.; Suri, J. Machine Learning in Medicine; CRC Press: Boca Raton, FL, USA, 2021.

16. Suri, J.S.; Rangayyan, R.M. Recent Advances in Breast Imaging, Mammography, and Computer-Aided Diagnosis of Breast Cancer; SPIE Press: Bellingham, WA, USA, 2006.

17. Biswas, M.; Kuppili, V.; Edla, D.R.; Suri, H.S.; Saba, L.; Marinhoe, R.T.; Sanches, J.M.; Suri, J.S. Symtosis: A liver ultrasound tissue characterization and risk stratification in optimized deep learning paradigm. Comput. Methods Programs Biomed. 2018, 155, 165-177. [CrossRef] [PubMed]

18. Acharya, U.R.; Sree, S.V.; Ribeiro, R.; Krishnamurthi, G.; Marinho, R.T.; Sanches, J.; Suri, J.S. Data mining framework for fatty liver disease classification in ultrasound: A hybrid feature extraction paradigm. Med. Phys. 2012, 39, 4255-4264. [CrossRef]

19. Acharya, U.R.; Sree, S.V.; Krishnan, M.M.R.; Molinari, F.; Garberoglio, R.; Suri, J.S. Non-invasive automated 3D thyroid lesion classification in ultrasound: A class of ThyroScan ${ }^{\mathrm{TM}}$ systems. Ultrasonics 2012, 52, 508-520. [CrossRef]

20. Acharya, U.R.; Swapna, G.; Sree, S.V.; Molinari, F.; Gupta, S.; Bardales, R.H.; Witkowska, A.; Suri, J.S. A review on ultrasoundbased thyroid cancer tissue characterization and automated classification. Technol. Cancer Res. Treat. 2014, 13, 289-301. [CrossRef] [PubMed]

21. Molinari, F.; Mantovani, A.; Deandrea, M.; Limone, P.; Garberoglio, R.; Suri, J.S. Characterization of single thyroid nodules by contrast-enhanced 3-D ultrasound. Ultrasound Med. Biol. 2010, 36, 1616-1625. [CrossRef] [PubMed]

22. Shrivastava, V.K.; Londhe, N.D.; Sonawane, R.S.; Suri, J.S. Computer-aided diagnosis of psoriasis skin images with HOS, texture and color features: A first comparative study of its kind. Comput. Methods Programs Biomed. 2016, 126, 98-109. [CrossRef] [PubMed]

23. Shrivastava, V.K.; Londhe, N.D.; Sonawane, R.S.; Suri, J.S. Reliable and accurate psoriasis disease classification in dermatology images using comprehensive feature space in machine learning paradigm. Expert Syst. Appl. 2015, 42, 6184-6195. [CrossRef]

24. Mookiah, M.R.K.; Acharya, U.R.; Martis, R.J.; Chua, C.K.; Lim, C.M.; Ng, E.; Laude, A. Evolutionary algorithm based classifier parameter tuning for automatic diabetic retinopathy grading: A hybrid feature extraction approach. Knowl. Based Syst. 2013, 39, 9-22. [CrossRef]

25. Than, J.C.; Saba, L.; Noor, N.M.; Rijal, O.M.; Kassim, R.M.; Yunus, A.; Suri, H.S.; Porcu, M.; Suri, J.S. Lung disease stratification using amalgamation of Riesz and Gabor transforms in machine learning framework. Comput. Biol. Med. 2017, 89, 197-211. [CrossRef] [PubMed]

26. Acharya, U.R.; Sree, S.V.; Krishnan, M.M.R.; Krishnananda, N.; Ranjan, S.; Umesh, P.; Suri, J.S. Automated classification of patients with coronary artery disease using grayscale features from left ventricle echocardiographic images. Comput. Methods Programs Biomed. 2013, 112, 624-632. [CrossRef]

27. Araki, T.; Ikeda, N.; Shukla, D.; Jain, P.K.; Londhe, N.D.; Shrivastava, V.K.; Banchhor, S.K.; Saba, L.; Nicolaides, A.; Shafique, S. PCA-based polling strategy in machine learning framework for coronary artery disease risk assessment in intravascular ultrasound: A link between carotid and coronary grayscale plaque morphology. Comput. Methods Programs Biomed. 2016, 128, 137-158. [CrossRef]

28. Acharya, R.U.; Faust, O.; Alvin, A.P.; Sree, S.V.; Molinari, F.; Saba, L.; Nicolaides, A.; Suri, J.S. Symptomatic vs. asymptomatic plaque classification in carotid ultrasound. J. Med. Syst. 2012, 36, 1861-1871. [CrossRef] [PubMed]

29. Acharya, U.R.; Faust, O.; Sree, S.V.; Molinari, F.; Saba, L.; Nicolaides, A.; Suri, J.S. An accurate and generalized approach to plaque characterization in 346 carotid ultrasound scans. IEEE Trans. Instrum. Meas. 2011, 61, 1045-1053. [CrossRef]

30. Suri, J.S. Imaging Based Symptomatic Classification and Cardiovascular Stroke Risk Score Estimation. Google Patent 13053971, 28 July 2011.

31. Cuadrado-Godia, E.; Dwivedi, P.; Sharma, S.; Ois Santiago, A.; Roquer Gonzalez, J.; Balcells, M.; Laird, J.; Turk, M.; Suri, H.S.; Nicolaides, A.; et al. Cerebral Small Vessel Disease: A Review Focusing on Pathophysiology, Biomarkers, and Machine Learning Strategies. J. Stroke 2018, 20, 302-320. [CrossRef]

32. Maniruzzaman, M.; Rahman, M.J.; Al-MehediHasan, M.; Suri, H.S.; Abedin, M.M.; El-Baz, A.; Suri, J.S. Accurate diabetes risk stratification using machine learning: Role of missing value and outliers. J. Med. Syst. 2018, 42, 1-17. [CrossRef] [PubMed]

33. Martis, R.J.; Acharya, U.R.; Prasad, H.; Chua, C.K.; Lim, C.M.; Suri, J.S. Application of higher order statistics for atrial arrhythmia classification. Biomed. Signal Process. Control. 2013, 8, 888-900. [CrossRef]

34. Suri, J.S.; Puvvula, A.; Biswas, M.; Majhail, M.; Saba, L.; Faa, G.; Singh, I.M.; Oberleitner, R.; Turk, M.; Chadha, P.S.; et al COVID-19 pathways for brain and heart injury in comorbidity patients: A role of medical imaging and artificial intelligence-based COVID severity classification: A review. Comput. Biol. Med. 2020, 124, 103960. [CrossRef] 
35. Suri, J.S.; Puvvula, A.; Majhail, M.; Biswas, M.; Jamthikar, A.D.; Saba, L.; Faa, G.; Singh, I.M.; Oberleitner, R.; Turk, M. Integration of cardiovascular risk assessment with COVID-19 using artificial intelligence. Rev. Cardiovasc. Med. 2020, 21, 541-560. [PubMed]

36. Agarwal, M.; Saba, L.; Gupta, S.K.; Carriero, A.; Falaschi, Z.; Paschè, A.; Danna, P.; El-Baz, A.; Naidu, S.; Suri, J.S. A novel block imaging technique using nine artificial intelligence models for COVID-19 disease classification, characterization and severity measurement in lung computed tomography scans on an Italian cohort. J. Med. Syst. 2021, 45, 1-30. [CrossRef]

37. Mansoor, A.; Bagci, U.; Foster, B.; Xu, Z.; Douglas, D.; Solomon, J.M.; Udupa, J.K.; Mollura, D.J. CIDI-lung-seg: A single-click annotation tool for automatic delineation of lungs from CT scans. In Proceedings of the 2014 36th Annual International Conference of the IEEE Engineering in Medicine and Biology Society, Chicago, IL, USA, 26-30 August 2014; pp. 1087-1090.

38. Saba, L.; Banchhor, S.K.; Araki, T.; Viskovic, K.; Londhe, N.D.; Laird, J.R.; Suri, H.S.; Suri, J.S. Intra- and inter-operator reproducibility of automated cloud-based carotid lumen diameter ultrasound measurement. Indian Heart J. 2018, 70, 649-664. [CrossRef]

39. Saba, L.; Banchhor, S.K.; Suri, H.S.; Londhe, N.D.; Araki, T.; Ikeda, N.; Viskovic, K.; Shafique, S.; Laird, J.R.; Gupta, A.; et al. Accurate cloud-based smart IMT measurement, its validation and stroke risk stratification in carotid ultrasound: A web-based point-of-care tool for multicenter clinical trial. Comput. Biol. Med. 2016, 75, 217-234. [CrossRef] [PubMed]

40. Saba, L.; Than, J.C.; Noor, N.M.; Rijal, O.M.; Kassim, R.M.; Yunus, A.; Ng, C.R.; Suri, J.S. Inter-observer Variability Analysis of Automatic Lung Delineation in Normal and Disease Patients. J. Med. Syst. 2016, 40, 142. [CrossRef]

41. Badrinarayanan, V.; Kendall, A.; Cipolla, R. SegNet: A Deep Convolutional Encoder-Decoder Architecture for Image Segmentation. IEEE Trans. Pattern Anal. Mach. Intell. 2017, 39, 2481-2495. [CrossRef]

42. Simonyan, K.; Zisserman, A. Very deep convolutional networks for large-scale image recognition. arXiv 2014, arXiv:1409.1556.

43. He, K.; Zhang, X.; Ren, S.; Sun, J. Deep residual learning for image recognition. In Proceedings of the IEEE Conference on Computer Vision and Pattern Recognition (CVPR); IEEE: Las Vegas, NV, USA, 2016; pp. 770-778.

44. Saba, L.; Banchhor, S.K.; Londhe, N.D.; Araki, T.; Laird, J.R.; Gupta, A.; Nicolaides, A.; Suri, J.S. Web-based accurate measurements of carotid lumen diameter and stenosis severity: An ultrasound-based clinical tool for stroke risk assessment during multicenter clinical trials. Comput. Biol. Med. 2017, 91, 306-317. [CrossRef]

45. Molinari, F.; Zeng, G.; Suri, J.S. Intima-media thickness: Setting a standard for a completely automated method of ultrasound measurement. IEEE Trans. Ultrason. Ferroelectr. Freq. Control. 2010, 57, 1112-1124. [CrossRef]

46. Suri, J.S.; Kathuria, C.; Molinari, F. Atherosclerosis Disease Management; Springer Science \& Business Media: New York, NY, USA, 2010.

47. Khanna, N.N.; Jamthikar, A.D.; Araki, T.; Gupta, D.; Piga, M.; Saba, L.; Carcassi, C.; Nicolaides, A.; Laird, J.R.; Suri, H.S.J.E Nonlinear model for the carotid artery disease 10-year risk prediction by fusing conventional cardiovascular factors to carotid ultrasound image phenotypes: A Japanese diabetes cohort study. Echocardiography 2019, 36, 345-361. [CrossRef]

48. Molinari, F.; Meiburger, K.M.; Saba, L.; Acharya, U.R.; Ledda, G.; Zeng, G.; Ho, S.Y.S.; Ahuja, A.T.; Ho, S.C.; Nicolaides, A.J.C.m.; et al. Ultrasound IMT measurement on a multi-ethnic and multi-institutional database: Our review and experience using four fully automated and one semi-automated methods. Comput. Methods Programs Biomed. 2012, 108, 946-960. [CrossRef] [PubMed]

49. Qualtrics, X. Determining Sample Size: How to Ensure You Get the Correct Sample Size. 2019. Available online: https: //www.qualtrics.com/au/experience-management/research/determine-sample-size/ (accessed on 28 July 2021).

50. Singh, B.K.; Verma, K.; Thoke, A.; Suri, J.S.J.M. Risk stratification of 2D ultrasound-based breast lesions using hybrid feature selection in machine learning paradigm. Measurement 2017, 105, 146-157. [CrossRef]

51. Riffenburgh, R.H.; Gillen, D.L. Contents. In Statistics in Medicine, 4th ed.; Academic Press: Cambridge, MA USA, 2020.

52. Priya, C.; Sithi Shameem Fathima, S.M.H.; Kirubanandasarathy, N.; Valanarasid, A.; Safana Begam, M.H.; Aiswarya, N. Automatic Optimized Cnn Based Covid-19 Lung Infection Segmentation from Ct Image. Mater. Today Proc. 2021. [CrossRef]

53. Saood, A.; Hatem, I. COVID-19 lung CT image segmentation using deep learning methods: U-Net versus SegNet. BMC Med. Imaging 2021, 21, 1-10. [CrossRef] [PubMed]

54. Paluru, N.; Dayal, A.; Jenssen, H.B.; Sakinis, T.; Cenkeramaddi, L.R.; Prakash, J.; Yalavarthy, P.K. Anam-Net: Anamorphic Depth Embedding-Based Lightweight CNN for Segmentation of Anomalies in COVID-19 Chest CT Images. IEEE Trans. Neural Netw. Learn. Syst. 2021, 32, 932-946. [CrossRef]

55. Cai, W.; Liu, T.; Xue, X.; Luo, G.; Wang, X.; Shen, Y.; Fang, Q.; Sheng, J.; Chen, F.; Liang, T. CT Quantification and Machine-learning Models for Assessment of Disease Severity and Prognosis of COVID-19 Patients. Acad. Radiol. 2020, 27, 1665-1678. [CrossRef] [PubMed]

56. Chaddad, A.; Hassan, L.; Desrosiers, C. Deep CNN models for predicting COVID-19 in CT and X-ray images. J. Med. Imaging 2021, 8, 014502. [CrossRef]

57. Gunraj, H.; Wang, L.; Wong, A. COVIDNet-CT: A Tailored Deep Convolutional Neural Network Design for Detection of COVID-19 Cases From Chest CT Images. Front. Med. 2020, 7, 608525. [CrossRef] [PubMed]

58. Iyer, T.J.; Joseph Raj, A.N.; Ghildiyal, S.; Nersisson, R. Performance analysis of lightweight CNN models to segment infectious lung tissues of COVID-19 cases from tomographic images. PeerJ Comput. Sci. 2021, 7, e368. [CrossRef]

59. Ranjbarzadeh, R.; Jafarzadeh Ghoushchi, S.; Bendechache, M.; Amirabadi, A.; Ab Rahman, M.N.; Baseri Saadi, S.; Aghamohammadi, A.; Kooshki Forooshani, M. Lung Infection Segmentation for COVID-19 Pneumonia Based on a Cascade Convolutional Network from CT Images. Biomed. Res. Int. 2021, 2021, 5544742. [CrossRef] [PubMed] 
60. Xiao, H.; Ran, Z.; Huang, J.; Ren, H.; Liu, C.; Zhang, B.; Dang, J. Research progress in lung parenchyma segmentation based on computed tomography. J. Biomed. Eng. 2021, 38, 379-386.

61. El-Kenawy, E.-S.M.; Ibrahim, A.; Mirjalili, S.; Eid, M.M.; Hussein, S.E. Novel feature selection and voting classifier algorithms for COVID-19 classification in CT images. IEEE Access 2020, 8, 179317-179335. [CrossRef]

62. Vijayalakshmi, A. Deep learning approach to detect malaria from microscopic images. Multimed. Tools Appl. 2020, 79, 15297-15317. [CrossRef]

63. Saba, L.; Sanagala, S.S.; Gupta, S.K.; Koppula, V.K.; Laird, J.R.; Viswanathan, V.; Sanches, M.J.; Kitas, G.D.; Johri, A.M.; Sharma, N. A Multicenter Study on Carotid Ultrasound Plaque Tissue Characterization and Classification Using Six Deep Artificial Intelligence Models: A Stroke Application. IEEE Trans. Instrum. Meas. 2021, 70, 1-12. [CrossRef]

64. Skandha, S.S.; Gupta, S.K.; Saba, L.; Koppula, V.K.; Johri, A.M.; Khanna, N.N.; Mavrogeni, S.; Laird, J.R.; Pareek, G.; Miner, M. 3-D optimized classification and characterization artificial intelligence paradigm for cardiovascular/stroke risk stratification using carotid ultrasound-based delineated plaque: Atheromatic ${ }^{\mathrm{TM}}$ 2.0. Comput. Biol. Med. 2020, 125, 103958. [CrossRef] [PubMed]

65. Kuppili, V.; Biswas, M.; Sreekumar, A.; Suri, H.S.; Saba, L.; Edla, D.R.; Marinhoe, R.T.; Sanches, J.M.; Suri, J.S. Extreme learning machine framework for risk stratification of fatty liver disease using ultrasound tissue characterization. J. Med. Syst. 2017, 41, 1-20. [CrossRef] [PubMed]

66. Jamthikar, A.; Gupta, D.; Khanna, N.N.; Saba, L.; Araki, T.; Viskovic, K.; Suri, H.S.; Gupta, A.; Mavrogeni, S.; Turk, M.J.C.d.; et al. A low-cost machine learning-based cardiovascular/stroke risk assessment system: Integration of conventional factors with image phenotypes. Cardiovasc. Diagn. Ther. 2019, 9, 420. [CrossRef]

67. El-Baz, A.; Suri, J.S. Big Data in Multimodal Medical Imaging; Boca Raton: CRC Press: Boca Raton, FL, USA, 2019.

68. Noor, N.M.; Than, J.C.; Rijal, O.M.; Kassim, R.M.; Yunus, A.; Zeki, A.A.; Anzidei, M.; Saba, L.; Suri, J.S. Automatic lung segmentation using control feedback system: Morphology and texture paradigm. J. Med. Syst. 2015, 39, 1-18. [CrossRef] [PubMed]

69. Jamthikar, A.; Gupta, D.; Saba, L.; Khanna, N.N.; Araki, T.; Viskovic, K.; Mavrogeni, S.; Laird, J.R.; Pareek, G.; Miner, M.J.C.D.; et al. Cardiovascular/stroke risk predictive calculators: A comparison between statistical and machine learning models. Cardiovasc. Diagn. Ther. 2020, 10, 919. [CrossRef] 